\title{
SUR LA DYNAMIQUE DE L'ÉLECTRON;
}

\author{
Par M. H. Poincaré (Paris).
}

Adunanza del 23 luglio rgos.

\section{INTRODUCTION.}

Il semble au premier abord que l'aberration de la lumière et les phénomènes optiques et électriques qui s'y rattachent vont nous fournir un moyen de déterminer le mouvement absolu de la Terre, ou plutôt son mouvement, non par rapport aux autres astres, mais par rapport à l'éther. FresNel l'avait déjà tenté, mais il reconnut bientôt que le mouvement de la Terre n'altère pas les lois de la réfraction et de la réflexion. Les expériences analogues, comme celle de la lunette pleine d'eau et toutes celles où on ne tient compte que des termes du $\mathrm{I}^{\text {er }}$ ordre par rapport à l'aberration, ne donnèrent non plus que des résultats négatifs; on en découvrit bientôt l'explication; mais Michelson, ayant imaginé une expérience où les termes dépendant du carré de l'aberration devenaient sensibles, échoua à son tour.

Il semble que cette impossibilité de mettre en évidence expérimentalement le mouvement absolu de la Terre soit une loi générale de la Nature; nous sommes naturellement portés à admettre cette loi, que nous appellerons le Postulat de Relativité et à l'admettre sans restriction. Que ce postulat, jusqu'ici d'accord avec l'expérience, doive être confirmé ou infirmé plus tard par des expériences plus précises, il est en tout cas intéressant de voir quelles en peuvent être les conséquences.

Une explication a été proposée par LoRentz et Fitz Gerald, qui ont introduit Phypothèse d'une contraction subie par tous les corps dans le sens du mouvement de la Terre et proportionnelle au carré de l'aberration; cette contraction, que nous appellerons la contraction lorentzienne, rendrait compte de l'expérience de Michelson et de toutes celles qui ont été réalisées jusqu'ici. L'hypothèse deviendrait insuffisante, toutefois, si on voulait admettre dans toute sa généralité le postulat de relativité.

LORENTz a cherché alors à la compléter et à la modifier de façon à la mettre en concordance parfaite avec ce postulat. C'est ce qu'il a réussi à faire dans 'son article intitule Electromagnetic phenomena in a system moving with any velocity smaller than that of light (Proceedings de l'Académie d'Amsterdam, 27 mai 1904).

L'importance de la question m'a déterminé à la reprendre; les résultats que j'ai 
obtenus sont d'accord avec ceux de M. LORENTz sur tous les points importants; j'ai été seulement conduit à les modifier et à les compléter dans quelques points de détail; on verra plus loin les différences qui sont d'une importance secondaire.

L'idée de Lorentz peut se résumer ainsi: si on peut, sans qu'aucun des phénomènes apparents soit modifié, imprimer à tout le système une translation commune, c'est que les équations d'un milieu électromagnétique ne sont pas altérées par certaines transformations, que nous appellerons transformations de LoRENTz; deux systèmes, l'un immobile, l'autre en translation, deviennent ainsi l'image exacte l'un de l'autre.

LANGEvin ${ }^{*}$ ) avait cherché à modifier l'idée de Lorentz; pour les deux auteurs, l'électron en mouvement prend la forme d'un ellipsoide aplati, mais pour Lorentz deux des axes de l'ellipsoïde demeurent constants, pour LANGEvin au contraire c'est le volume de l'ellipsoïde qui demeure constant. Les deux savants ont d'ailleurs montré que ces deux hypothèses s'accordent avec les expériences de Kaufmann, aussi bien que l'hypothèse primitive d'Abraham (électron sphérique indéformable).

L'avantage de la théorie de Langevin, c'est qu'elle ne fait intervenir que les forces électromagnétiques et les forces de liaison; mais elle est incompatible avec le postulat de relativité; c'est ce que LoRENTz avait montré, c'est ce que je retrouve à mon tour par une autre voie en faisant appel aux principes de la théorie des groupes.

Il faut donc en revenir à la théorie de LoRentz; mais si l'on veut la conserver et éviter d'intolérables contradictions, il faut supposer une force spéciale qui explique à la fois la contraction et la constance de deux des axes. J'ai cherché à déterminer cette force, j'ai trouvé qu'elle peut être assimilée à une pression extérieure constante, agissant sur l'electron déformable et compressible, et dont le travail est proportionnel aux variations du volume de cet électron.

Si alors l'inertie de la matière était exclusivement d'origine électromagnétique, comme on l'admet généralement depuis l'expérience de KaUfmann, et qu'à part cette pression constante dont je viens de parler, toutes les forces soient d'origine électromagnétique, le postulat de relativité peut être établi en toute rigueur. C'est ce que je montre par un calcul très simple fondé sur le principe de moindre action.

Mais ce n'est pas tout. Lorentz, dans l'ouvrage cité, a jugé nécessaire de compléter son hypothèse de façon à ce que le postulat subsiste quand il $\mathrm{y}$ a d'autres forces que les forces électromagnétiques. D'après lui, toutes les forces, quelle qu'en soit l'origine, sont affectées par la transformation de LORENTz (et par conséquent par une translation) de la même manière que les forces électromagnétiques.

Il importait d'examiner cette hypothèse de plus près et en particulier de rechercher quelles modifications elle nous obligerait a apporter aux lois de la gravitation.

On trouve d'abord qu'elle nous force à supposer que la propagation de la gravi-

*) Langevin avait été devancé par M. Bucherer de Bonn, qui a émis avant lui la même idée. (Voir: Bucherer, Mathematische Einführung in die Elektronentheorie; août I904. Teubner, Leipzig). 
tation n'est pas instantanée, mais se fait avec la vitesse de la lumière. On pourrait croire que c'est une raison suffisante pour rejeter l'hypothèse, LAPLACE ayant démontré qu'il ne peut en être ainsi. Mais en réalité, l'effet de cette propagation est compensé, en grande partie, par une cause différente, de sorte qu'il n'y a plus contradiction entre la loi proposee et les observations astronomiques.

Était-il possible de trouver une loi, qui satisfit à la condition imposée par LoREnTz, et qui en même temps se réduisit à la loi de Newton toutes les fois que les vitesses des astres sont assez petites pour qu'on puisse négliger leurs carrés (ainsi que le produit des accélerations par les distances) devant le carré de la vitesse de la Lumière?

A cette question, ainsi qu'on le verra plus loin, on doit répondre affirmativement.

La loi ainsi modifiée est-elle compatible avec les observations astronomiques?

A première vue, il semble que oui, mais la question ne pourra être tranchée que par une discussion approfondie.

Mais en admettant même que cette discussion tourne à l'avantage de la nouvelle hypothèse, que devrons-nous conclure? Si la propagation de l'attraction se fait avec la vitesse de la lumière, cela ne peut être par une rencontre fortuite, cela doit être parce que c'est une fonction de l'éther; et alors il faudra chercher a pénétrer la nature de cette fonction, et la rattacher aux autres fonctions du fluide.

Nous ne pouvons nous contenter de formules simplement juxtaposées et qui ne s'accorderaient que par un hasard heureux; il faut que ces formules arrivent pour ainsi dire à se pénétrer mutuellement. L'esprit ne sera satisfait que quand il croira apercevoir la raison de cet accord, au point d'avoir l'illusion qu'il aurait pu le prévoir.

Mais la question peut encore se présenter $d$ un autre point de vue, qu'une comparaison fera mieux comprendre. Supposons un astronome antérieur à CoPERNIC et réfléchissant sur le système de PToLÉmée; il remarquera que pour toutes les planètes, un des deux cercles, épicycle ou déférent, est parcouru dans le même temps. Cela ne peut être par hasard, il y a donc entre toutes les planètes je ne sais quel lien mystérieux.

Mais Copernic, en changeant simplement les axes de coordonnées regardés comme fixes, fait évanouir cette apparence; chaque planète ne décrit plus qu'un seul cercle et les durées des révolutions deviennent indépendantes (jusqu'à ce que KePLER rétablisse entre elles le lien qu'on avait cru détruit).

Ici il est possible qu'il y ait quelque chose d'analogue; si nous admettions le postulat de relativité, nous trouverions dans la loi de gravitation et dans les lois électromagnétiques un nombre commun qui serait la vitesse de la lumière; et nous le retrouverions encore dans toutes les autres forces d'origine quelconque, ce qui ne pourrait s'expliquer que de deux manières:

Ou bien il n'y aurait rien au monde qui ne fût d'origine électromagnétique.

Ou bien cette partie qui serait pour ainsi dire commune d tous les phénomènes physiques ne serait qu'une apparence, quelque chose qui tiendrait à nos méthodes de 
mesure. Comment faisons-nous nos mesures? En trasportant, les uns sur les autres, des objets regardés comme des solides invariables, répondra-t-on d'abord; mais cela n'est plus vrai dans la théorie actuelle, si l'on admet la contraction lorentzienne. Dans cette théorie, deux longueurs égales, ce sont, par définition, deux longueurs que la lumière met le même temps à parcourir.

Peut-être suffirait-il de renoncer ì cette définition, pour que la théorie de LoRENTz fût aussi complètement bouleversée que l'a été le système de Ptolémée par l'intervention de Copernic. Si cela arrive un jour, cela ne prouvera pas que l'effort fait par LoRentz ait été inutile; car Ptolémée, quoi qu'on en pense, n'a pas été inutile à COPERNIC.

Aussi n'ai-je pas hésité à publier ces quelques résultats partiels, bien qu'en ce moment même la théorie entière puisse sembler mise en danger par la découverte des rayons magnétocathodiques.

\section{$\int$ I. - Transformation de Lorentz.}

LORENTZ a adopté un système particulier d'unités, de façon à faire disparaitre les facteurs $4 \pi$ dans les formules. Je ferai de même, et de plus je choisirai les unités de longueur et de temps de telle façon que la vitesse de la lumière soit égale à $\mathbf{r}$. Dans ces conditions les formules fondamentales deviennent, en appelant $f, g, b$ le déplacement électrique, $\alpha, \xi, \gamma$ la force magnétique, $F, G, H$ le potentiel vecteur, $\psi$ le potentiel scalaire, $\rho$ la densité électrique, $\xi, n, \zeta$ la vitesse de l'electron, $u, v, w$ le courant :

$$
\left\{\begin{array}{c}
u=\frac{d f}{d t}+\rho \xi=\frac{d \gamma}{d y}-\frac{d \beta}{d z}, \quad \alpha=\frac{d H}{d y}-\frac{d G}{d z}, f=-\frac{d F}{d t}-\frac{d \psi}{d x}, \\
\frac{d \alpha}{d t}=\frac{d g}{d z}-\frac{d b}{d y}, \quad \frac{d \rho}{d t}+\sum \frac{d \rho \xi}{d x}=0, \quad \sum \frac{d f}{d x}=\rho, \quad \frac{d \psi}{d t}+\sum \frac{d F}{d x}=0, \\
\square=\Delta-\frac{d^{2}}{d t^{2}}=\sum \frac{d^{2}}{d x^{2}}-\frac{d^{2}}{d t^{2}}, \quad \square \psi=-\rho, \quad \square F=-\rho \xi .
\end{array}\right.
$$

Un élément de matière de volume $d x d y d z$, subit une force mécanique dont les composantes $X d x d y d z, Y d x d y d z, Z d x d y d z$ se déduisent de la formule:

$$
X=p f+\rho(n \gamma-\zeta \beta) \text {. }
$$

Ces équations sont susceptibles d'une transformation remarquable découverte par LoRENTZ et qui doit son intérêt à ce qu'elle explique pourquoi aucune expérience n'est susceptible de nous faire connaitre le mouvement absolu de l'univers. Posons :

$$
x^{\prime}=k l(x+\varepsilon t), \quad t^{\prime}=k l(t+\varepsilon x), \quad y^{\prime}=l y, \quad z^{\prime}=l z,
$$

$l$ et $\varepsilon$ étant deux constantes quelconques, et étant

$$
k=\frac{\mathrm{I}}{\sqrt{\mathrm{I}-\varepsilon^{2}}} \text {. }
$$


Si alors nous posons:

il viendra:

$$
\square^{\prime}=\sum \frac{d^{2}}{d x^{\prime 2}}-\frac{d^{2}}{d t^{\prime 2}}
$$

$$
\square^{\prime}=\square l^{-2}
$$

Considérons une sphère entrainée avec l'électron dans un mouvement de translation uniforme et soit :

$$
(x-\xi t)^{2}+(y-n t)^{2}+(z-\zeta t)^{2}=r^{2}
$$

l'équation de cette sphère mobile dont le volume sera $\frac{4}{3} \pi r^{2}$.

La transformation la changera en un ellipsoide, dont il est aisé de trouver l'equation. On déduit aisément en effet des équations (3):

$$
\left(3^{\mathrm{bis}}\right) \quad x=\frac{k}{l}\left(x^{\prime}-\varepsilon t^{\prime}\right), \quad t=\frac{k}{l}\left(t^{\prime}-\varepsilon x^{\prime}\right), \quad y=\frac{y^{\prime}}{l}, \quad z=\frac{z^{\prime}}{i} .
$$

L'équation de l'ellipsoïde devient ainsi :

$k^{2}\left(x^{\prime}-\varepsilon t^{\prime}-\xi t^{\prime}+\varepsilon \xi x^{\prime}\right)^{2}+\left(y^{\prime}-n k t^{\prime}+n k \varepsilon x^{\prime}\right)^{2}+\left(z^{\prime}-\zeta k t^{\prime}+\zeta k \varepsilon x^{\prime}\right)^{2}=l^{2} r^{2}$.

Cet ellipsoïde se déplace avec un mouvement uniforme; pour $t^{\prime}=0$, il se réduit $\mathrm{a}$

$$
k^{2} x^{\prime 2}(\mathrm{r}+\zeta \varepsilon)^{2}+\left(y^{\prime}+n k \varepsilon x^{\prime}\right)^{2}+\left(z^{\prime}+\zeta k \varepsilon x^{\prime}\right)^{2}=l^{2} r^{2}
$$

et a pour volume :

$$
\frac{4}{3} \pi r^{3} \frac{l^{3}}{k(\mathrm{I}+\xi \varepsilon)} .
$$

Si l'on veut que la charge d'un électron ne soit pas altérée par la transformation et si l'on appelle $\rho^{\prime}$ la nouvelle densité électrique, il viendra :

$$
\rho^{\prime}=\frac{k}{l^{3}}(\rho+\varepsilon \rho \xi)
$$

Que seront maintenant les nouvelles vitesses $\xi^{\prime}, n^{\prime}, \zeta^{\prime}$; on devra avoir:

$$
\begin{gathered}
\xi^{\prime}=\frac{d x^{\prime}}{d t^{\prime}}=\frac{d(x+\varepsilon t)}{d(t+\varepsilon x)}=\frac{\xi+\varepsilon}{\mathrm{I}+\varepsilon \xi}, \\
n^{\prime}=\frac{d y^{\prime}}{d t^{\prime}}=\frac{d y}{k d(t+\varepsilon x)}=\frac{n}{k(\mathrm{I}+\varepsilon \xi)}, \quad \zeta^{\prime}=\frac{\zeta}{k(\mathrm{I}+\varepsilon \xi)},
\end{gathered}
$$

d'où :

$\left(4^{\text {bis }}\right) \quad p^{\prime} \xi^{\prime}=\frac{k}{l^{3}}(p \xi+\varepsilon p), \quad p^{\prime} n^{\prime}=\frac{I}{l^{3}} p^{\prime}, \quad p^{\prime} \zeta^{\prime}=\frac{I}{l^{3}} p \zeta$.

C'est ici que je dois signaler pour la première fois une divergence avec LoRENTz.

LORENTz pose (a la différence des notations près) (loco citato, page 813, formules 7 et 8 ):

$$
p^{\prime}=\frac{1}{k l^{3}} p, \quad \xi^{\prime}=k^{2}(\xi+\varepsilon), \quad n^{\prime}=k n, \quad \zeta^{\prime}=k \zeta .
$$


On retrouve ainsi les formules:

$$
\rho^{\prime} \xi^{\prime}=\frac{k}{l^{3}}(\rho \xi+\varepsilon \rho), \quad \rho^{\prime} n^{\prime}=\frac{1}{l^{3}} \rho r_{1}, \quad \rho^{\prime} \zeta^{\prime}=\frac{1}{l^{3}} \rho \zeta ;
$$

mais la valeur de $p^{\prime}$ diffère.

Il importe de remarquer que les formules (4) et $\left(4^{\text {bis }}\right)$ satisfont à la condition de continuité

$$
\frac{d \rho^{\prime}}{d t^{\prime}}+\sum \frac{d \rho^{\prime} \xi^{\prime}}{d x^{\prime}}=0
$$

Soit en effet $\lambda$ une quantité indétermin'e et $D$ le déterminant fonctionnel de

$$
t+\lambda_{\rho}, \quad x+\lambda_{\rho} \xi, \quad y+\lambda_{\rho} n, \quad z+\lambda_{\rho} \zeta
$$

par rapport à $t, x, y, z$. On aura:

$$
D=D_{\mathrm{o}}+D_{\mathrm{r}} \lambda+D_{2} \lambda^{2}+D_{3} \lambda^{3}+D_{4} \lambda^{4} \text {. }
$$

$\operatorname{avec} D_{\mathrm{o}}=\mathrm{I}, D_{\mathrm{r}}=\frac{d \rho}{d t}+\sum \frac{d \rho \xi}{d x}=0$.

Soit $\lambda^{\prime}=l^{2} \lambda$, nous voyons que les 4 fonctions

$\left(5^{\text {bis }}\right) \quad t^{\prime}+\lambda^{\prime} \rho^{\prime}, \quad x^{\prime}+\lambda^{\prime} \rho^{\prime} \xi^{\prime}, \quad y^{\prime}+\lambda^{\prime} \rho^{\prime} n^{\prime}, \quad z^{\prime}+\lambda^{\prime} \rho^{\prime} \zeta^{\prime}$

sont liées aux fonctions $(\zeta)$ par les mêmes relations linéaires que les variables anciennes aux variables nouvelles. $\mathrm{Si}$ donc on désigne par $D^{\prime}$ le déterminant fonctionnel des fonctions $\left(5^{\text {bis }}\right)$ par rapport aux variables nouvelles, on aura:

d'où :

$$
D^{\prime}=D, \quad D^{\prime}=D_{\mathrm{o}}^{\prime}+D_{\mathrm{s}}^{\prime} \lambda^{\prime}+\cdots+D_{4}^{\prime} \lambda^{\prime 4},
$$

$$
D_{\mathrm{o}}^{\prime}=D_{\mathrm{o}}=\mathrm{I}, \quad D_{\mathrm{r}}^{\prime}=l^{-2} D_{\mathrm{r}}=0=\frac{d \rho^{\prime}}{d t^{\prime}}+\sum \frac{d \rho^{\prime} \xi^{\prime}}{d x^{\prime}} . \quad \text { C. Q. F. D. }
$$

Avec l'hypothèse de LoRENTz, cette condition ne serait pas remplie, puisque $\rho$ ' n'a pas la même valeur.

Nous définirons les nouveaux potentiels, vecteur et scalaire, de façon à satisfaire aux conditions

$$
\square^{\prime} \psi^{\prime}=-\rho^{\prime}, \quad \square F^{\prime}=-\rho^{\prime} \xi^{\prime} .
$$

Nous tirerons ensuite de lat :

(7) $\psi^{\prime}=\frac{k}{l}(\psi+\varepsilon F), \quad F^{\prime}=\frac{k}{l}(F+\varepsilon \psi), \quad G^{\prime}=\frac{\mathrm{I}}{l} G, \quad H^{\prime}=\frac{\mathrm{I}}{l} H$.

Ces formules diffèrent notablement de celles de LOREntz, mais la divergence ne porte en dernière analyse que sur les définitions.

Nous choisirons les nouveaux champs électrique et magnétique de façon à satisfaire aux équations :

$$
f^{\prime}=-\frac{d F^{\prime}}{d t^{\prime}}-\frac{d \psi^{\prime}}{d x^{\prime}}, \quad \alpha^{\prime}=\frac{d H^{\prime}}{d y^{\prime}}-\frac{d G^{\prime}}{d z^{\prime}} .
$$


Il est aisé de voir que:

$\frac{d}{d t^{\prime}}=\frac{k}{l}\left(\frac{d}{d t}-\varepsilon \frac{d}{d x}\right), \quad \frac{d}{d x^{\prime}}=\frac{k}{l}\left(\frac{d}{d x}-\varepsilon \frac{d}{d t}\right), \frac{d}{d y^{\prime}}=\frac{\mathrm{I}}{l} \frac{d}{d y}, \frac{d}{d z^{\prime}}=\frac{\mathrm{I}}{l} \frac{d}{d z}$ et on en conclut :

(9)

$$
\left\{\begin{array}{lll}
f^{\prime}=\frac{\mathrm{I}}{l^{2}} f, & g^{\prime}=\frac{k}{l^{2}}(g+\varepsilon \gamma), & b^{\prime}=\frac{k}{l^{2}}(b-\varepsilon \beta), \\
\alpha^{\prime}=\frac{\mathrm{I}}{l^{2}} \alpha, & \beta^{\prime}=\frac{k}{l^{2}}(\beta-\varepsilon b), & \gamma^{\prime}=\frac{k}{l^{2}}(\gamma+\varepsilon g) .
\end{array}\right.
$$

Ces formules sont identiques à celles de Lorentz.

Notre transformation n'altère pas les équations (I). En effet, la condition de continuité, ainsi que les équations (6) et (8), nous fournissent déjà quelques-unes des équations (I) (sauf l'accentuation des lettres).

Les équations (6) rapprochées de la condition de continuité donnent:

Il reste à établir que:

$$
\frac{d \psi^{\prime}}{d t^{\prime}}+\sum \frac{d F^{\prime}}{d x^{\prime}}=0
$$

$$
\frac{d f^{\prime}}{d t^{\prime}}+\rho^{\prime} \xi^{\prime}=\frac{d \vartheta^{\prime}}{d y^{\prime}}-\frac{d \xi^{\prime}}{d z^{\prime}}, \quad \frac{d x^{\prime}}{d t^{\prime}}=\frac{d g^{\prime}}{d z^{\prime}}-\frac{d b^{\prime}}{d y^{\prime}}, \quad \sum \frac{d f^{\prime}}{d x^{\prime}}=\xi^{\prime}
$$

et l'on voit aisément que ce sont des conséquences nécessaires des équations (6), (8) et (10).

Nous devons maintenant comparer les forces avant et après la transformation.

Soient $X, Y, Z$ la force avant, et $X^{\prime}, Y^{\prime}, Z^{\prime}$ la force après la transformation, toutes deux rapportées à.l'unité de volume. Pour que $X^{\prime}$ satisfasse aux mêmes équations qu'avant la transformation, on doit avoir:

$$
\begin{aligned}
& X^{\prime}=\rho^{\prime} f^{\prime}+\rho^{\prime}\left(n^{\prime} \gamma^{\prime}-\zeta^{\prime} \beta^{\prime}\right), \\
& Y^{\prime}=\rho^{\prime} g^{\prime}+\rho^{\prime}\left(\zeta^{\prime} \alpha^{\prime}-\xi^{\prime} \gamma^{\prime}\right), \\
& Z^{\prime}=\rho^{\prime} b^{\prime}+\rho^{\prime}\left(\xi^{\prime} \beta^{\prime}-n^{\prime} \alpha^{\prime}\right),
\end{aligned}
$$

ou, en remplaçant toutes les quantités par leurs valeurs $(4),\left(4^{\text {bis }}\right)$ et $(9)$ et tenant compte des équations (2):

$$
\left\{\begin{array}{l}
X^{\prime}=\frac{k}{l^{5}}\left(X+\varepsilon \sum X \xi\right), \\
Y^{\prime}=\frac{1}{l^{5}} Y \\
Z^{\prime}=\frac{\mathrm{I}}{l^{5}} Z .
\end{array}\right.
$$

Si nous représentions par $X_{1}, Y_{1}, Z_{1}$ les composantes de la force rapportie, non plus à l'unité de volume, mais à l'unité de charge électrique de l'électron, et par $X_{1}^{\prime}$, $Y_{\mathrm{r}}^{\prime}, Z_{1}^{\prime}$ les mêmes quantités après la transformation, nous aurions:

$$
X_{1}=f+n \gamma-\zeta \beta, \quad X_{1}^{\prime}=f^{\prime}+n^{\prime} \gamma^{\prime}-\zeta^{\prime} \beta^{\prime}, \quad X=\rho X_{1}, \quad X^{\prime}=\rho^{\prime} X_{\mathrm{r}}^{\prime}
$$


et nous aurions les équations :

(II $\left.{ }^{\text {bis }}\right)$

$$
\left\{\begin{array}{l}
X_{1}^{\prime}=\frac{k}{l^{5}} \frac{\rho}{\rho^{\prime}}\left(X_{1}+\varepsilon \sum X_{\mathrm{r}} \xi\right) \\
Y_{\mathrm{r}}^{\prime}=\frac{\mathrm{I}}{l^{5}} \frac{\rho}{\rho^{\prime}} Y_{1}, \\
Z_{\mathrm{s}}^{\prime}=\frac{\mathrm{I}}{l^{5}} \frac{\rho}{\rho^{\prime}} Z_{\mathrm{r}} .
\end{array}\right.
$$

LORENTz avait trouvé [à la différence des notations près, page $8 \mathbf{I}_{3}$, formule (io)]:

$\left(I^{\text {ter }}\right)$

$$
\left\{\begin{array}{l}
X_{\mathrm{r}}=l^{2} X_{\mathrm{1}}^{\prime}-l^{2} \varepsilon\left(n^{\prime} g^{\prime}+\zeta^{\prime} b^{\prime}\right), \\
Y_{\mathrm{I}}=\frac{l^{2}}{k} Y_{\mathrm{r}}^{\prime}+\frac{l^{2} \varepsilon}{k} \xi^{\prime} g^{\prime}, \\
Z_{\mathrm{I}}=\frac{l^{2}}{k} Z_{\mathrm{r}}^{\prime}+\frac{l^{2} \varepsilon}{k} \xi^{\prime} b^{\prime} .
\end{array}\right.
$$

Avant d'aller plus loin, il importe de rechercher la cause de cette importante divergence. Elle tient évidemment a ce que les formules pour $\xi^{\prime}, n^{\prime}, \zeta^{\prime}$ ne sont pas les mêmes, tandis que les formules pour les champs électriques et magnétiques sont les mêmes.

Si l'inertie des électrons est exclusivement d'origine électromagnétique, si de plus ils ne sont soumis qu'à des forces d'origine électromagnétique, la condition d'équilibre exige que l'on ait à l'intérieur des électrons:

$$
X=Y=Z=\text { o. }
$$

Or en vertu des équations (I I) ces relations équivalent à

$$
X^{\prime}=Y^{\prime}=Z^{\prime}=0 \text {. }
$$

Les conditions d'équilibre des électrons ne sont donc pas altérées par la transformation.

Malheureusement une hypothèse aussi simple est inadmissible. Si, en effet, on suppose $\zeta=n=\zeta=0$, les conditions $X=Y=Z=0$ entraineraient $f=g=b=0$, et par conséquent $\sum \frac{d f}{d x}=0$, c'est-à-dire $\rho=0$. On arriverait à des résultats analogues dans le cas le plus général. Il faut donc bien admettre qu'il y a outre les forces électromagnétiques, soit d'autres forces, soit des liaisons. Il faut alors chercher à quelles conditions doivent satisfaire ces forces ou ces liaisons, pour que l'equilibre des électrons ne soit pas troublé par la transformation. Ce sera l'objet d'un paragraphe ultérieur.

\section{2. - Principe de moindre action.}

On sait comment Lorentz a déduit ses équations du principe de moindre action. Je reviendrai cependant sur la question, bien que je n'aie rien d'essentiel à ajouter d̀ l'analyse de LORENTz, parce que je préfère la présenter sous une forme un peu différente qui me sera utile pour mon objet. Je poserai:

$$
J=\int d t d \tau\left[\frac{\sum f^{2}}{2}+\frac{\sum \alpha^{2}}{2}-\sum F u\right]
$$


en supposant que $f, \alpha, F, u$, etc. sont assujetties aux conditions suivantes et à celles qu'on en déduirait par symétrie :

$$
\sum \frac{d f}{d x}=\rho, \quad \alpha=\frac{d H}{d y}-\frac{d G}{d z}, \quad u=\frac{d f}{d t}+\rho \xi .
$$

Quant à l'intégrale $J$ elle doit être étendue :

$I^{\circ}$ par rapport ì l'élément de volume $d \tau=d x d y d z$, à l'espace tout entier;

$2^{\circ}$ par rapport au temps $t$, à l'intervalle compris entre les limites $t=t_{\mathrm{o}}, t=t_{\mathrm{I}}$.

D'après le principe de moindre action, l'intégrale $J$ doit être un minimum, si l'on assujettit les diverses quantités qui y figurent:

$I^{\circ}$ aux conditions (2);

$2^{\circ}$ à la condition que l'état du système soit déterminé aux deux époques limites $t=t_{\mathrm{o}}, t=t_{\mathrm{s}}$.

Cette dernière condition nous permet de transformer nos intégrales par intégration par parties par rapport au temps. Si nous avons en effet une intégrale de la forme

$$
\int d t d \tau A \frac{d B \delta C}{d t}
$$

où $C$ est une des quantités qui définissent l'état du système et $\delta C$ sa variation, elle sera égale (en intégrant par parties par rapport au temps):

$$
\int d \tau|A B \delta C|_{\substack{t=t_{\mathrm{o}} \\ t=t_{\mathrm{I}}}}-\int d t d \tau \frac{d A}{d t} d B \delta C .
$$

Comme l'état du système est déterminé aux deux époques limites, on a $\delta C=0$ pour $t=t_{\mathrm{o}}, t=t_{\mathrm{s}}$; donc la $\mathrm{I}^{\text {ère }}$ intégrale qui se rapporte à ces deux époques est nulle, et la $2^{\text {de }}$ subsiste seule.

Nous pouvons de même intégrer par parties par rapport d $x, y$ ou $z$; nous avons en effet

$$
\int A \frac{d B}{d x} d x d y d z d t=\int A B d y d z d t-\int B \frac{d A}{d x} d x d y d z d t
$$

Nos intégrations s'étendant jusqu'à l'infini, il faut faire $x= \pm \infty$ dans la I ìre intégrale du $2^{\text {de }}$ membre; donc, comme nous supposons toujours que toutes nos fonctions s'annulent à l'infini, cette intégrale sera nulle et il viendra

$$
\int A \frac{d B}{d} d \tau d t=-\int B \frac{d A}{d x} d \tau d t
$$

Si le système était supposé soumis à des liaisons, il faudrait adjoindre ces conditions de liaison aux conditions imposées aux diverses quantités qui figurent dans l'intégrale $J$.

Donnons d'abord à $F, G, H$ des accroissements $\delta F, \delta G, \delta H$; d'où :

$$
\delta \alpha=\frac{d \delta H}{d y}-\frac{d \delta G}{d z} .
$$


On devra avoir

$$
\delta J=\int d t d \tau\left[\sum \alpha\left(\frac{d \delta H}{d y}-\frac{d \delta G}{d z}\right)-\sum u \delta F\right]=0,
$$

ou, en intégrant par parties,

$$
\delta J=\int d t d \tau\left[\sum\left(\delta G_{\overline{d z}}^{d x}-\delta H \frac{d x}{d y}\right)-\sum u \delta F\right]=-\int d t d \tau \sum \delta F\left(u-\frac{d \gamma}{d y}+\frac{d \beta}{d z}\right)=0,
$$

d'où, en égalant à zéro le coefficient de l'arbitraire $\delta F$,

$$
u=\frac{d \gamma}{d y}-\frac{d \beta}{d z} .
$$

Cette relation nous donne (avec une intégration par parties):

$$
\begin{gathered}
\int \sum F u d \tau=\int \sum F\left(\frac{d \gamma}{d y}-\frac{d \beta}{d z}\right) d \tau=\int \sum\left(\beta \frac{d F}{d z}-\gamma \frac{d F}{d y}\right) d \tau=\int \sum \alpha\left(\frac{d H}{d y}-\frac{d G}{d z}\right) d \tau, \\
\int \sum F u d \tau=\int \sum \alpha^{2} d \tau,
\end{gathered}
$$

d'où enfin :

$$
J=\int d t d \tau\left(\frac{\sum f^{2}}{2}-\frac{\sum \alpha^{2}}{2}\right) \text {. }
$$

Désormais, et grâce d̀ la relation (3), $\delta J$ est indépendant de $\delta F$ et par conséquent de $\delta \alpha$; faisons varier maintenant les autres variables.

Il vient, en revenant à l'expression (I) de $J$,

$$
\delta J=\int d t d \tau\left(\sum f \delta f-\sum F \delta u\right) .
$$

Mais $f, g, b$ sont assujettis à la $\mathrm{I}^{\text {ère }}$ des conditions (2), de sorte que

$$
\sum \frac{d \delta f}{d x}=\delta \rho
$$

et qu'il convient d'écrire :

$$
\delta J=\int d t d \tau\left[\sum f d f-\sum F \delta u-\psi\left(\sum \frac{d \delta f}{d x}-\delta \rho\right)\right] .
$$

Les principes du calcul des variations nous apprennent que l'on doit faire le calcul comme si, $\psi$ étant une fonction arbitraire, $\delta J$ était représenté par l'expression (6) et si les variations n'étaient plus assujetties à la condition ( 5 ).

Nous avons d'autre part

$$
\delta u=\frac{d \delta f}{d t}+\delta \rho \xi
$$

d'où, après intégration par parties,

$$
\delta J=\int d t d \tau \sum \delta f\left(f+\frac{d F}{d t}+\frac{d \psi}{d x}\right)+\int d t d \tau\left(\psi \delta \rho-\sum F \delta \rho \xi\right) .
$$

Si nous supposons d'abord que les électrons ne subissent pas de variation, 
$\delta_{\rho}=\delta \rho \xi=o$ et la seconde intégrale est nulle. Comme $\delta J$ doit s'annuler, on doit avoir :

$$
f+\frac{d F}{d t}+\frac{d \psi}{d x}=0
$$

Il reste donc dans le cas général:

$$
\delta J=\int d t d \tau\left(\psi \delta \rho-\sum F \delta \rho \xi\right) .
$$

Il reste à déterminer les forces qui agissent sur les électrons. Pour cela nous devons supposer qu'on applique à chaque élément d'électron une force complémentaire $-X d \tau,-Y d \tau,-Z d \tau$ et écrire que cette force fait équilibre aux forces d'origine électromagnétique. Soit $U, V, W$ les composantes du déplacement de l'élément $d \tau$ d'électron, déplacement compté à partir d'une position initiale quelconque. Soient $\delta U$, $\delta V, \delta W$ les variations de ce déplacement; le travail virtuel correspondant de la force complémentaire sera:

$$
-\int \sum X \delta U d \tau
$$

de sorte que la condition d'équilibre dont nous venons de parler s'écrira:

$$
\delta J=-\int \sum X \delta U d \tau d t .
$$

Il s'agit de transformer $\delta J$. Pour cela commençons par chercher l'équation de continuité exprimant que la charge d'un électron se conserve par la variation.

Soient $x_{0}, y_{0}, z_{0}$ la position initiale d'un électron. Sa position actuelle sera:

$$
x=x_{0}+U, \quad y=y_{0}+V, \quad z=z_{0}+W .
$$

Nous introduirons en outre une variable auxiliaire $\varepsilon$, qui produira les variations de nos diverses fonctions, de sorte que, pour une fonction $A$ quelconque, on ait:

$$
\delta A=\delta \varepsilon \frac{d A}{d \varepsilon} \text {. }
$$

Il me sera commode en effet de pouvoir passer de la notation du calcul des variations, à celle du calcul differentiel ordinaire, ou inversement.

Nos fonctions pourront être regardées: $\mathrm{I}^{\circ}$ soit comme dépendant des cinq variables $x, y, z, t, \varepsilon$, de telle sorte qu'on reste toujours à la même place quand $t$ et $\varepsilon$ varient seuls: nous désignerons alors leurs dérivées par des $d$ ordinaires; $2^{\circ}$ soit comme dépendant des cinq variables $x_{0}, y_{0}, z_{0}, t, \varepsilon$, de telle sorte qu'on suive toujours un même électron quand $t$ et $\varepsilon$ varient seuls: nous désignerons alors leurs dérivées par des $\partial$ ronds. On aura alors :

$$
\xi=\frac{\partial U}{\partial t}=\frac{d U}{d t}+\xi \frac{d U}{d x}+n \frac{d U}{d y}+\zeta \frac{d U}{d z}=\frac{\partial x}{\partial t} .
$$

Désignons maintenant per $\Delta$ le déterminant fonctionnel de $x, y, z$ par rapport d 
$x_{0}, y_{0}, z_{0}:$

$$
\Delta=\frac{\partial(x, y, z)}{\partial\left(x_{0}, y_{0}, z_{0}\right)}
$$

Si $\varepsilon, x_{0}, y_{0}, z_{0}$ restant constants nous donnons à $t$ un accroissement $\partial t$, il en résultera pour $x, y, z$ des accroissements $\partial x, \partial y, \partial z$, et pour $\Delta$ un accroissement $\partial \Delta$, et on aura:

d'oú

$$
\begin{aligned}
& \partial x=\xi \partial t, \quad \partial y=n \partial t, \quad \partial z=\zeta \partial t, \\
& \Delta+\partial \Delta=\frac{\partial(x+\partial x, y+\partial y, z+\partial z)}{\partial\left(x_{0}, y_{0}, z_{0}\right)}
\end{aligned}
$$

$$
\mathrm{I}+\frac{\partial \Delta}{\Delta}=\frac{\partial(x+\partial x, y+\partial y, z+\partial z)}{\partial(x, y, z)}=\frac{\partial(x+\xi \partial t, y+n \partial t, z+\zeta \partial t)}{\partial(x, y, z)} .
$$

On en déduit:

$$
\frac{\mathrm{I}}{\Delta} \frac{\partial \Delta}{\partial t}=\frac{d \xi}{d x}+\frac{d \eta}{d y}+\frac{d \zeta}{d z} .
$$

La masse de chaque électron étant invariable, on aura:

$$
\frac{\partial p \Delta}{\partial t}=0
$$

d'où :

$$
\frac{\partial \rho}{\partial t}+\sum \rho \frac{d \xi}{d x}=0, \quad \frac{\partial \rho}{\partial t}=\frac{d \rho}{d t}+\sum \xi \frac{d \rho}{d x}, \quad \frac{d \rho}{d t}+\sum \frac{d \rho \xi}{d x}=0
$$

Telles sont les différentes formes de l'équation de continuité en ce qui concerne la variable $t$. Nous trouvons des formes analogues en ce qui concerne la variable $\varepsilon$. Soit :

il viendra:

$$
\delta U=\frac{\partial U}{\partial \varepsilon} \delta \varepsilon, \quad \delta V=\frac{\partial V}{\partial \varepsilon} \delta \varepsilon, \quad \delta W=\frac{\partial W}{\partial \varepsilon} \delta \varepsilon
$$

(I ${ }^{\text {bis }}$ )

$$
\delta U=\frac{d U}{d \varepsilon} \delta \varepsilon+\delta U \frac{d U}{d x}+\delta V \frac{d U}{d y}+\delta W \frac{d U}{d z}
$$

$\left(12^{\text {bis }}\right)$

$$
\frac{\mathrm{I}}{\Delta} \frac{\partial \Delta}{\partial \varepsilon}=\sum \frac{\partial U}{\partial \varepsilon}, \quad \frac{\partial p \Delta}{\partial \varepsilon}=0
$$

$\left(\mathrm{I} 3^{\text {bis }}\right) \delta \varepsilon \frac{\partial \rho}{\partial \varepsilon}+\sum \rho \frac{d \delta U}{d x}=0, \quad \frac{\partial p}{\partial \varepsilon}=\frac{d \rho}{d \varepsilon}+\sum \frac{\delta U}{\delta \varepsilon} \frac{d \rho}{d x}, \quad \delta \rho+\frac{d \rho \delta U}{d x}=0$.

On remarquera la difference entre la définition de $\delta U=\frac{\partial U}{\partial \varepsilon} \delta \varepsilon$ et celle de $\delta \rho=\frac{d \rho}{d \varepsilon} \delta \varepsilon$; on remarquera que c'est bien cette définition de $\delta U$ qui convient a la formule (I0).

Cette dernière équation va nous permettre de trasformer le $I^{\text {er }}$ terme de (9); nous trouvons en effet :

$$
\int d t d \tau \psi \delta \rho=-\int d t d \tau \psi \sum \frac{d \rho \delta U}{d x}
$$


ou, en intégrant par parties,

$$
\int d t d \tau \psi \delta \rho=\int d t d \tau \sum \rho \frac{d \psi}{d x} \delta U
$$

Proposons-nous maintenant de déterminer

$$
\delta(\rho \xi)=\frac{d(\rho \xi)}{d \varepsilon} \delta \varepsilon .
$$

Observons que $\rho \Delta$ ne peut dépendre que de $x_{0}, y_{0}, z_{0}$; en effet, si l'on consi lère un élément d'électron dont la position initiale est un parallélipipède rectangle dont les arêtes sont $d x_{0}, d y_{0}, d z_{0}$, la charge de cet élément est

$$
p \Delta d x_{0} d y_{0} d z_{0}
$$

et, cette charge devant demeurer constante, on a:

On en déduit:

$$
\frac{\partial \rho \Delta}{\partial t}=\frac{\partial \rho \Delta}{\partial \varepsilon}=0
$$

$$
\frac{\partial^{2} p \Delta U}{\partial t \partial \varepsilon}=\frac{\partial}{\partial \varepsilon}\left(\rho \Delta \frac{\partial U}{\partial t}\right)=\frac{\partial}{\partial t}\left(\rho \Delta \frac{\partial U}{\partial \varepsilon}\right) .
$$

Or on sait que pour une fonction $A$ quelconque on a, par l'équation de continuité,

et de même

$$
\frac{\mathrm{I}}{\Delta} \frac{\partial A \Delta}{\partial t}=\frac{d A}{d t}+\sum \frac{d A \xi}{d x}
$$

On a donc:

$$
\frac{\mathrm{I}}{\Delta} \frac{\partial A \Delta}{\partial s}=\frac{d A}{d s}+\sum \frac{d A \frac{\partial U}{\partial \varepsilon}}{d x}
$$

$$
\begin{aligned}
& \text { (17) } \frac{\mathrm{I}}{\Delta} \frac{\partial}{\partial \varepsilon}\left(\rho \Delta \frac{\partial U}{\partial t}\right)=\frac{d \rho \frac{\partial U}{\partial t}}{\partial \varepsilon}+\frac{d\left(\rho \frac{\partial U}{\partial t} \frac{\partial U}{\partial \varepsilon}\right)}{d x}+\frac{d\left(\rho \frac{\partial U}{\partial t} \frac{\partial V}{\partial \varepsilon}\right)}{d y}+\frac{d\left(\rho \frac{\partial U}{\partial t} \frac{\partial W}{\partial \varepsilon}\right)}{d z} \\
& \left(\mathrm{I}^{\text {bis }}\right) \frac{\mathrm{I}}{\Delta} \frac{\partial}{\partial t}\left(\rho \Delta \frac{\partial U}{\partial \varepsilon}\right)=\frac{d \rho \frac{\partial U}{\partial \varepsilon}}{d t}+\frac{d\left(\rho \frac{\partial U}{\partial t} \frac{\partial U}{\partial \varepsilon}\right)}{d x}+\frac{d\left(\rho \frac{\partial V}{\partial t} \frac{\partial U}{\partial \varepsilon}\right)}{d y}+\frac{d\left(\rho \frac{\partial W}{\partial t} \frac{\partial U}{\partial \varepsilon}\right)}{d z} .
\end{aligned}
$$

Les $2^{\text {ds }}$ membres de (I7) et ( $17^{\text {bis }}$ ) doivent être égaux et, si l'on se souvient que

il vient :

$$
\frac{\partial U}{\partial t}=\xi, \quad \frac{\partial U}{\partial \varepsilon} \delta \varepsilon=\delta U, \quad \frac{d \rho \xi}{d \varepsilon} \delta \varepsilon=\delta \rho \xi,
$$

$$
\delta \rho \xi+\frac{d(\rho \xi \delta U)}{d x}+\frac{d(\rho \xi \delta V)}{d y}+\frac{d(\rho \xi \delta W)}{d z}=\frac{d(\rho \delta U)}{d t}+\frac{d(\rho \xi \delta U)}{d x}+\frac{d(\rho n \delta U)}{d y}+\frac{d(\rho \zeta \delta U)}{d z}
$$

Transformons maintenant le $2^{d}$ terme de (9); il vient:

$$
\begin{gathered}
\int d t d \tau \sum F \delta \rho \xi \\
=\int d t d \tau\left[\sum F \frac{d(\rho \delta U)}{d t}+\sum F \frac{d(\rho \eta \delta U)}{d y}+\sum F \frac{d(\rho \zeta \delta U)}{d z}-\sum F \frac{d(\rho \xi \delta V)}{d y}-\sum F \frac{d(\rho \xi \delta W)}{d z}\right] .
\end{gathered}
$$


Le second membre devient, par l'intégration par parties:

$$
\int d t d \tau\left[-\sum \rho \delta U \frac{d F}{d t}-\sum \rho \eta \delta U \frac{d F}{d y}-\sum \rho \zeta \delta U \frac{d F}{d z}+\sum \rho \xi \delta V \frac{d F}{d y}+\sum \rho \xi \delta W \frac{d F}{d z}\right] \text {. }
$$

Remarquons maintenant que:

$$
\sum \rho \xi \delta V \frac{d F}{d y}=\sum \rho \zeta \delta U \frac{d H}{d x}, \quad \sum \rho \xi \delta W \frac{d F}{d z}=\sum \rho \eta \delta U \frac{d G}{d x} .
$$

$\mathrm{Si}$, en effet, dans les deux membres de ces relations, on développe les $\sum$, elles deviennent des identités; et souvenons-nous que

$$
\frac{d H}{d x}-\frac{d F}{d z}=-\beta, \quad \frac{d G}{d x}-\frac{d F}{d y}=\gamma,
$$

le second membre en question deviendra:

$$
\int d t d \tau\left[-\sum \rho \delta U \frac{d F}{d t}+\sum \rho \gamma \eta \delta U-\sum \rho \beta \zeta \delta U\right],
$$

de sorte que finalement:

$\delta J=\int d t d \tau \sum p^{\delta} U\left(\frac{d \psi}{d x}+\frac{d F}{d t}+\beta \zeta-\gamma n\right)=\int d t d \tau \sum \rho \delta U(-f+\beta \zeta-\gamma n)$.

En égalant le coefficient de $\delta U$ dans les deux membres de (10) il vient:

$$
X=f-\beta \zeta+\gamma^{n} \text {. }
$$

C'est l'équation (2) du $\$ précédent.

\section{$\int 3 .-$ La transformation de Lorentz et le principe de moindre action.}

Voyons si le principe de moindre action nous donne la raison du succès de la transformation de LORENTz. Il faut d'abord voir ce que cette transformation fait de l'intégrale:

(formule $4 \mathrm{du} \ 2$ ).

$$
J=\int d t d \tau\left(\frac{\sum f^{2}}{2}-\frac{\sum \alpha^{2}}{2}\right)
$$

Nous trouvons d'abord

$$
d t^{\prime} d \tau^{\prime}=l^{+} d t d \tau
$$

car $x^{\prime}, y^{\prime}, z^{\prime}, t^{\prime}$ sont liés à $x, y, z, t$ par des relations linéaires dont le déterminant est égal à $l^{4}$; il vient ensuite:

$$
\left\{\begin{array}{l}
l^{4} \sum f^{2}=f^{2}+k^{2}\left(g^{2}+b^{2}\right)+k^{2} \varepsilon^{2}\left(\beta^{2}+\gamma^{2}\right)+2 k^{2} \varepsilon(g \gamma-b \beta) \\
l^{4} \sum \alpha^{\prime 2}=\alpha^{2}+k^{2}\left(\beta^{2}+\gamma^{2}\right)+k^{2} \varepsilon^{2}\left(g^{2}+b^{2}\right)+2 k^{2} \varepsilon(g \gamma-b \beta)
\end{array}\right.
$$

(formules $9 \mathrm{du} \ \mathrm{I}$ ), d'où:

$$
l^{4}\left(\sum f^{\prime 2}-\sum \alpha^{\prime 2}\right)=\sum f^{2}-\sum \alpha^{2}
$$


de sorte que si l'on pose:

il vient:

$$
J^{\prime}=\int d t^{\prime} d \tau^{\prime}\left(\frac{\sum f^{\prime 2}}{2}-\frac{\sum \alpha^{\prime 2}}{2}\right)
$$

$$
J^{\prime}=J \text {. }
$$

Il faut toutefois, pour que cette égalité soit justifiée, que les limites d'intégration soient les mêmes; jusqu'ici nous avons admis que $t$ variait depuis $t_{\mathrm{o}}$ jusqu'à $t_{1}$, et $x$, $y, z$ depuis $-\infty$ jusqu'd $+\infty$. A ce compte les limites d'intégration seraient altérées par la transformation de LoRentz; mais rien ne nous empêche de supposer $t_{0}=-\infty$, $t_{\mathrm{x}}=+\infty$; avec ces conditions les limites sont les mêmes pour $J$ et pour $J^{\prime}$.

Nous avons alors a comparer les deux équations suivantes analogues d l'équation (I0) du $\ 2$ :

$$
\left\{\begin{array}{l}
\delta J=-\int \sum X \delta U d \tau d t \\
\delta J^{\prime}=-\int \sum X^{\prime} \delta U^{\prime} d \tau^{\prime} d t^{\prime} .
\end{array}\right.
$$

Pour cela, il faut d'abord comparer $\delta U^{\prime}$ à $\delta U$.

Considérons un électron dont les coordonnées initiales sont $x_{0}, y_{0}, z_{0}$; ses coordonnées à l'instant $t$, seront

$$
x=x_{0}+U, \quad y=y_{0}+V, \quad z=z_{0}+W .
$$

$\mathrm{Si}$ on considère l'électron correspondant après la transformation de LoRENTz, il aura pour coordonnées

où

$$
x^{\prime}=k l(x+\varepsilon t), \quad y^{\prime}=l y, \quad z^{\prime}=l z,
$$

$$
x^{\prime}=x_{0}+U^{\prime}, \quad y^{\prime}=y_{0}+V^{\prime}, \quad z^{\prime}=z_{0}+W^{\prime} ;
$$

mais il n'atteindra ces coordonnćes qu'd l'instant

$$
t^{\prime}=k l(t+\varepsilon x) \text {. }
$$

Si nous faisons subir à nos variables des variations $\delta U, \delta V, \delta W$ et que nous donnions en même temps à $t$ un accroissement $\delta t$, les coordonnées $x, y$, z subiront un accroissement total

$$
\delta x=\delta U+\xi \delta t, \quad \delta y=\delta V+n \delta t, \quad \delta z=\delta W+\zeta \delta t .
$$

Nous aurons de même:

$$
\delta x^{\prime}=\delta U^{\prime}+\xi^{\prime} \delta t^{\prime}, \quad \delta y^{\prime}=\delta V^{\prime}+n^{\prime} \delta t^{\prime}, \quad \delta z^{\prime}=\delta W^{\prime}+\zeta^{\prime} \delta t^{\prime}
$$

et en vertu de la transformation de LoRENTz:

$$
\delta x^{\prime}=k l(\delta x+\varepsilon \delta t), \quad \delta y^{\prime}=l \delta y, \quad \delta z^{\prime}=l \delta z, \quad \delta t^{\prime}=k t(\delta t+\varepsilon \delta x),
$$

d'où, en supposant $\delta t=0$, les relations:

$$
\begin{aligned}
& \delta x^{\prime}=\delta U^{\prime}+\xi^{\prime} \delta t^{\prime}=k l \delta U, \\
& \delta y^{\prime}=\delta V^{\prime}+n^{\prime} \delta t^{\prime}=l \delta V, \\
& \delta t^{\prime}=k l_{\varepsilon} \delta U .
\end{aligned}
$$


Remarquons que

$$
\xi^{\prime}=\frac{\xi+\varepsilon}{I+\xi_{\varepsilon}}, \quad n^{\prime}=\frac{n}{k(1+\xi \varepsilon)} ;
$$

il vi'ndra, en remplaçant $\delta t^{\prime}$ par sa valeur,

$$
\begin{aligned}
k l(\mathrm{I}+\xi \varepsilon) \delta U & =\delta U^{\prime}(\mathrm{I}+\xi \varepsilon)+(\xi+\varepsilon) k l \varepsilon \delta U, \\
l(\mathrm{I}+\xi \varepsilon) \delta V & =\delta V^{\prime}(\mathrm{I}+\xi \varepsilon)+n l \varepsilon \delta U .
\end{aligned}
$$

Si nous nous rappelons la définition de $k$, nous tirerons de là :

et de même

$$
\begin{aligned}
& \delta U=\frac{k}{l} \delta U^{\prime}+\frac{k \varepsilon}{l} \xi \delta U^{\prime}, \\
& \delta V=\frac{1}{l} \delta V^{\prime}+\frac{k \varepsilon}{l} n \delta U^{\prime},
\end{aligned}
$$

d'où

$$
\delta W=\frac{\mathrm{I}}{l} \delta W^{\prime}+\frac{k \varepsilon}{l} \zeta \delta U^{\prime}
$$

(3) $\sum X \delta U=\frac{1}{l}\left(k X \delta U^{\prime}+Y \delta V^{\prime}+Z \delta W^{\prime}\right)+\frac{k \varepsilon}{l} \delta U^{\prime} \sum X \xi$.

Or, en vertu des équations (2) on doit avoir:

$$
\int \sum X^{\prime} \delta U^{\prime} d t^{\prime} d \tau^{\prime}=\int \sum X \delta U d t d \tau=\frac{\mathrm{I}}{l^{4}} \int \sum X \delta U d t^{\prime} d \tau^{\prime} .
$$

En remplaçant $\sum X \delta U$ par sa valeur (3) et identifiant, il vient:

$$
X^{\prime}=\frac{k}{l^{5}} X+\frac{k \varepsilon}{l^{5}} \sum X \xi, \quad Y^{\prime}=\frac{\mathrm{I}}{l^{5}} Y, \quad Z^{\prime}=\frac{\mathrm{I}}{l^{5}} Z .
$$

Ce sont les équations (II) du $\$ I. Le principe de moindre action nous conduit don: au même résultat que l'analyse du $\ \mathrm{I}$.

Si nous nous reportons aux formules (I), nous voyons que $\sum f^{2}-\sum \alpha^{2}$ n'est pas altérée par la transformation de LoRENTz, sauf un facteur constant; il n'en est pas de nnême de l'expression $\sum f^{2}+\sum \alpha^{2}$ qui figure dans l'énergie. Si nous nous bornons au cas où $\varepsilon$ est assez petit pour qu'on en puisse négliger le carré de sorte que $k=: \mathrm{I}$ et si nous supposons aussi $l=\mathrm{I}$, nous trouvons:

ou, par addition,

$$
\begin{aligned}
& \sum f^{\prime 2}=\sum f^{2}+2 \varepsilon(g \gamma-b \beta), \\
& \sum \alpha^{\prime 2}=\sum \alpha^{2}+2 \varepsilon(g \gamma-b \beta),
\end{aligned}
$$

$$
\sum f^{\prime 2}+\sum \alpha^{\prime 2}=\sum f^{2}+\sum \alpha^{2}+4 \varepsilon(g \gamma-b \beta) .
$$

\section{\4. - Le Groupe de Lorentz.}

Il importe de remarquer que les transformations de LORENTz forment un groupe. Si l'on pose en effet:

$$
x^{\prime}=k l(x+\varepsilon t), \quad y^{\prime}=l y, \quad z^{\prime}=l k, \quad t^{\prime}=k l(t+\varepsilon x),
$$


et d'autre part

$$
x^{\prime \prime}=k^{\prime} l^{\prime}\left(x^{\prime}+\varepsilon^{\prime} t^{\prime}\right), \quad y^{\prime \prime}=l^{\prime} y^{\prime}, \quad z^{\prime \prime}=l^{\prime} z^{\prime}, \quad t^{\prime \prime}=k^{\prime} l^{\prime}\left(t^{\prime}+\varepsilon^{\prime} x^{\prime}\right),
$$

avec

il viendra:

$$
k^{-2}=\mathrm{I}-\varepsilon^{2}, \quad k^{-2}=\mathrm{I}-\varepsilon^{\prime 2},
$$

$$
x^{\prime \prime}=k^{\prime \prime} l^{\prime \prime}\left(x+\varepsilon^{\prime \prime} t\right), \quad y^{\prime \prime}=l^{\prime \prime} y, \quad z^{\prime \prime}=l^{\prime \prime} z, \quad t^{\prime \prime}=k^{\prime \prime} l^{\prime \prime}\left(t+\varepsilon^{\prime \prime} x\right),
$$

avec

$$
\varepsilon^{\prime \prime}=\frac{\varepsilon+\varepsilon^{\prime}}{1+\varepsilon \varepsilon^{\prime}}, \quad l^{\prime \prime}=l l^{\prime}, \quad k^{\prime \prime}=k k^{\prime}\left(\mathrm{I}+\varepsilon \varepsilon^{\prime}\right)=\frac{\mathrm{I}}{\sqrt{\mathrm{I}-\varepsilon^{\prime \prime 2}}} .
$$

Si nous donnons à $l$ la valeur $\mathrm{I}$, que nous supposions $\varepsilon$ infiniment petit,

$$
x^{\prime}=x+\delta x, \quad y^{\prime}=y+\delta y, \quad z^{\prime}=z+\delta z, \quad t^{\prime}=t+\delta t,
$$

il viendra:

$$
\delta x=\varepsilon t, \quad \delta y=\delta z=0, \quad \delta t=\varepsilon x .
$$

C'est là la transformation infinitésimale génératrice du groupe, que j’appellerai la transformation $\dot{T}_{\text {s }}$ et qui d'après la notation de LIE peut s'écrire:

$$
t \frac{d \varphi}{d x}+x \frac{d \varphi}{d t}=T_{i}
$$

Si nous supposons $\varepsilon=0$ et $l=\mathrm{I}+\delta l$, nous trouverions au contraire

$$
\delta x=x \delta l, \quad \delta y=y \delta l, \quad \delta z=z \delta l, \quad \delta t=t \delta l .
$$

et nous aurions une autre transformation infinitésimale $T_{\mathrm{o}}$ du groupe (a supposer que $l$ et $\varepsilon$ soient regardés comme des variables indépendantes) et on aurait avec la notation de LIE :

$$
T_{\mathrm{o}}=x \frac{d \varphi}{d x}+y \frac{d \varphi}{d y}+z \frac{d \varphi}{d z}+t \frac{d \varphi}{d t} .
$$

Mais on pourrait faire jouer à l'axe des $y$ ou à celui des $z$ le rôle particulier que nous avons fait jouer a l'axe des $x$; on aurait ainsi deux autres transformations infinitésimales :

$$
\begin{aligned}
& T_{2}=t \frac{d \varphi}{d y}+y \frac{d \varphi}{d t}, \\
& T_{3}=t \frac{d \varphi}{d z}+z \frac{d \varphi}{d t},
\end{aligned}
$$

qui n'altéreraient pas non plus les équations de Lorentz.

On peut former les combinaisons imaginées par LIE, telles que

$$
\left[T_{\mathrm{r}}, T_{2}\right]=x \frac{d \varphi}{d y}-y \frac{d \varphi}{d x}
$$

mais il est aisé de voir que cette transformation équivaut à un changement d'axes de coordonnées, les axes tournant d'un angle très petit autour de l'axe des $z$. Nous ne 
devons donc pas nous étonner si un pareil changement n'altère pas la forme des équations de LORENTz, évidemment indépendantes du choix des axes.

Nous sommes donc amenés à envisager un groupe continu que nous appellerons le groupe de LORENTZ et qui admettra comme transformations infinitésimales:

$I^{\circ}$ la transformation $T_{0}$ qui sera permutable à toutes les autres;

$2^{\circ}$ les trois transformations $T_{1}, T_{2}, T_{3}$;

$3^{\circ}$ les trois rotations $\left[T_{1}, T_{2}\right],\left[T_{2}, T_{3}\right],\left[T_{3}, T_{1}\right]$.

Une transformation quelconque de ce groupe pourra toujours se décomposer en une transformation de la forme:

$$
x^{\prime}=l x, \quad y^{\prime}=l y, \quad z^{\prime}=l z, \quad t^{\prime}=l t
$$

et une transformation linéaire qui n'altère pas la forme quadratique

$$
x^{2}+y^{2}+z^{2}-t^{2} \text {. }
$$

Nous pouvons encore engendrer notre groupe d'une autre manière. Toute transformation du groupe pourra être regardée comme une transformation de la forme:

$$
x^{\prime}=k l(x+\varepsilon t), \quad y^{\prime}=l y, \quad z^{\prime}=l z, \quad t^{\prime}=k l(t+\varepsilon x)
$$

précédée et suivie d'une rotation convenable.

Mais pour notre objet, nous ne devons considérer qu'une partie des transformations de ce groupe; nous devons supposer que $l$ est une fonction de $\varepsilon$, et il s'agit de choisir cette fonction, de façon que cette partie du groupe, que j'appellerai $P$ forme encore un groupe.

Faisons tourner le système de $180^{\circ}$ autour de l'axe des $y$, nous devrons retrouver une transformation qui devra encore appartenir à $P$. Or cela revient à changer le signe de $x, x^{\prime}, z$ et $z^{\prime}$; on trouve ainsi:

$$
x^{\prime}=k l(x-\varepsilon t), \quad y^{\prime}=l y, \quad z^{\prime}=l \tilde{k}, \quad t^{\prime}=k l(t-\varepsilon x) .
$$

Donc $l$ ne change pas quand on change $\varepsilon$ en $-\varepsilon$.

D'autre part, si $P$ est un groupe, la substitution inverse de (I), qui s'écrit:

$$
x^{\prime}=\frac{k}{l}(x-\varepsilon t), \quad y^{\prime}=\frac{y}{l}, \quad z^{\prime}=\frac{z}{l}, \quad t^{\prime}=\frac{k}{l}(t-\varepsilon x),
$$

devra également appartenir à $P$; elle devra donc être identique d̀ (2), c'est-d̀-dire que

$$
l=\frac{\mathrm{I}}{l} \text {. }
$$

On devra donc avoir $l=\mathrm{I}$.

\section{5. - Ondes de Langevin.}

M. Langevin a mis sous une forme particulièrement élégante les formules qui définissent le champ électromagnétique produit par le mouvement d'un électron unique.

Reprenons les équations

$$
\square \psi=-\rho, \quad \square F=-\rho \xi .
$$


On sait qu'on peut les intégrer par les potentiels retardés et qu'on $a$ :

$$
\psi=\frac{\mathrm{I}}{4 \pi} \int \frac{\underline{p}_{1} d \tau_{1}}{r}, \quad F=\frac{\mathrm{I}}{4 \pi} \int \frac{p_{\mathrm{I}} \xi_{\mathrm{I}} d \tau_{\mathrm{I}}}{r} .
$$

Dans ces formules on a :

$$
d \tau_{\mathrm{r}}=d x_{\mathrm{r}} d y_{\mathrm{I}} d z_{\mathrm{I}}, \quad r^{2}=\left(x-x_{1}\right)^{2}+\left(y-y_{\mathrm{x}}\right)^{2}+\left(z-z_{\mathrm{I}}\right)^{2},
$$

tandis que $\rho_{1}$ et $\xi_{1}$ sont les valeurs de $\rho$ et de $\xi$ au point $x_{1}, y_{I}, z_{1}$ et à l'instant

$$
t_{\mathrm{r}}=t-r \text {. }
$$

Soient: $x_{0}, y_{0}, z_{0}$ les coordonnées d'une molécule d'électron à l'instant $t$;

$$
x_{\mathrm{s}}=x_{\mathrm{o}}+U, \quad y_{\mathrm{s}}=y_{\mathrm{o}}+V, \quad z_{\mathrm{r}}=z_{\mathrm{o}}+W
$$

ses coordonnées d̀ linstant $t_{\mathrm{r}}$.

$U, V, W$ sont des fonctions de $x_{0}, y_{0}, z_{0}$, de sorte que nous pourrons écrire:

$$
d x_{1}=d x_{0}+\frac{d U}{d x_{0}} d x_{0}+\frac{d U}{d y_{0}} d y_{0}+\frac{d U}{d z_{0}} d z_{0}+\xi_{1} d t_{1} ;
$$

et si l'on suppose $t$ constant, ainsi que $x, y$ et $z$ :

$$
d t_{\mathrm{t}}=+\sum \frac{x-x_{\mathrm{r}}}{r} d x_{\mathrm{r}} .
$$

Nous pouvons done écrire:

$d x_{1}\left(\mathrm{I}+\xi_{1} \frac{x_{1}-x}{r}\right)+d y_{\mathrm{r}} \xi_{1} \frac{y_{1}-y}{r}+d z_{\mathrm{r}} \xi_{\mathrm{I}} \frac{z_{\mathrm{r}}-z}{r}=d x_{\mathrm{o}}\left(\mathrm{I}+\frac{d U}{d x_{0}}\right)+d y_{\mathrm{o}} \frac{d U}{d y_{0}}+d z_{0} \frac{d U}{d z_{0}}$ avec les deux autres équations qu'on peut en déduire par permutation circulaire.

Nous avons donc:

(3) $d \tau_{\mathrm{I}}\left|\mathrm{I}+\xi_{\mathrm{I}} \frac{x_{\mathrm{I}}-x}{r}, \quad \xi_{\mathrm{I}} \frac{y_{1}-y}{r}, \quad \xi_{1} \frac{z_{1}-z}{r}\right|=d \tau_{0}\left|\mathrm{I}+\frac{d U}{d x_{0}}, \frac{d U}{d} \frac{U}{y_{0}}, \frac{d U}{d z_{0}}\right|$, en posant

$$
d \tau_{\mathrm{o}}=d x_{\mathrm{o}} d y_{\mathrm{o}} d z_{\mathrm{o}} .
$$

Étudions les déterminants qui figurent dans les deux membres de (3) et d'abord dans le $\mathrm{I}^{\text {er }}$ membre; si on cherche $a$ le développer, on voit que les termes du $2^{\mathrm{d}}$ et du $3^{\mathrm{e}}$ degré par rapport $\mathrm{d} \xi_{1}, n_{1}, \zeta_{1}$ disparaissent et que le déterminant est égal à

$$
\mathrm{I}+\xi_{1} \frac{x_{\mathrm{I}}-x}{r}+n_{1} \frac{y_{1}-\underline{y}}{r}+\zeta_{1} \frac{z_{\mathrm{I}}-z}{r}=\mathrm{I}+\omega,
$$

w désignant la composante radiale de la vitesse $\xi_{1}, n_{1}, \zeta_{1}$, c'est-d-dire la composante dirigée suivant le rayon vecteur qui va du point $x, y, z$ au point $x_{1}, y_{\mathrm{s}}, z_{\mathrm{I}}$.

Pour obtenir le $2^{\mathrm{d}}$ déterminant, j'envisage les coordonnées des différentes molécules de l'électron à un instant $t_{1}^{\prime}$ qui est le même pour toutes les molécules, mais de telle façon que pour la molécule que $j$ 'envisage on ait $t_{1}=t_{1}^{\prime}$. Les coordonnées d'une molécule seront alors:

$$
x_{\mathrm{r}}^{\prime}=x_{\mathrm{o}}+U^{\prime}, \quad y_{\mathrm{s}}^{\prime}=y_{\mathrm{o}}+V^{\prime}, \quad z_{\mathrm{I}}^{\prime}=z_{\mathrm{o}}+W^{\prime},
$$


$U^{\prime}, V^{\prime}, W^{\prime}$ étant ce que deviennent $U, V, W$ quand on y remplace $t_{1}$ par $t_{1}^{\prime}$; comme $t_{\mathrm{r}}^{\prime}$ est le même pour toutes les molécules, on aura:

et par conséquent

$$
d x_{\mathrm{r}}^{\prime}=d x_{\mathrm{o}}\left(\mathrm{I}+\frac{d U^{\prime}}{d x_{\mathrm{o}}}\right)+d y_{\mathrm{o}} \frac{d U^{\prime}}{d y_{\mathrm{o}}}+d z_{\mathrm{o}} \frac{d U^{\prime}}{d z_{\mathrm{o}}}
$$

en posant

$$
d \tau_{1}^{\prime}=d \tau_{0}\left|\mathrm{I}+\frac{d U^{\prime}}{d x_{\mathrm{o}}}, \frac{d U^{\prime}}{d y_{\mathrm{o}}}, \frac{d U^{\prime}}{d z_{\mathrm{o}}}\right| \text {, }
$$

$$
d \tau_{\mathrm{I}}^{\prime}=d x_{\mathrm{I}}^{\prime} d y_{\mathrm{r}}^{\prime} d z_{\mathrm{I}}^{\prime} \text {. }
$$

Mais l'élément de charge électrique est

$$
d \mu_{\mathrm{s}}=p_{\mathrm{s}} d \tau_{\mathrm{s}}^{\prime}
$$

et de plus pour la molécule envisagée, on a $t_{1}=t_{1}^{\prime}$ et par conséquent $\frac{d U^{\prime}}{d x_{0}}=\frac{d U}{d x_{0}}$, etc.; nous pouvons donc écrire:

$$
d \mu_{\mathrm{I}}=p_{\mathrm{I}} d \tau_{\mathrm{o}}\left|\mathrm{I}+\frac{d U}{d x_{\mathrm{o}}}, \frac{d U}{d y_{\mathrm{o}}}, \frac{d U}{d z_{\mathrm{o}}}\right|,
$$

de sorte que l'équation (3) deviendra:

et les équations (2):

$$
p_{1} d \tau_{1}(I+\omega)=d \mu_{1}
$$

$$
\psi=\frac{\mathrm{I}}{4 \pi} \int \frac{d \mu_{\mathrm{I}}}{r(\mathrm{I}+\omega)}, \quad F=\frac{\mathrm{I}}{4 \pi} \int \frac{\xi_{\mathrm{I}} d \mu_{\mathrm{x}}}{r(\mathrm{I}+\omega)} .
$$

$\mathrm{Si}$ nous avons affaire à un électron unique, nos intégrales se réduiront d̀ un seul élément, pourvu que l'on ne considère que des points $x, y, z$ suffisamment éloignés pour que $r$ et $\omega$ aient sensiblement la même valeur pour tous les points de l'électron. Les potentiels $\psi, F, G, H$ dépendront de la position de cet électron, et aussi de sa vitesse, car non seulement $\xi_{\mathrm{I}}, n_{\mathrm{t}}, \zeta_{\mathrm{t}}$ figurent au numérateur dans $F, G, H$, mais la composante radiale $\omega$ figure au dénominateur. Il s'agit bien entendu de sa position et de sa vitesse $a$ l'instant $t_{1}$.

Les dérivées partielles de $\psi, F, G, H$ par rapport à $t, x, y, z$ (et par conséquent les champs électrique et magnétique) dépendront en outre de son accélération. De plus, elles en dépendront linéairement, puisque dans ces dérivées cette accélération s'introduit par suite d'une différentiation unique.

LANGEVIN a été ainsi conduit à distinguer dans les champs électrique et magnétique les termes qui ne dépendent pas de l'accélération (c'est ce qu'il appelle l'onde de vitesse) et ceux qui sont proportionnels à l'accélération (c'est ce qu'il appelle l'onde d'accélération).

Le calcul de ces deux ondes est facilité par la transformation de LoRENTz. Nous pouvons en effet appliquer cette transformation au système, de façon que la vitesse de l'électron unique envisagé devienne nulle. Nous prendrons pour l'axe des $x$ la direction de cette vitesse avant la transformation, de sorte que, a l'instant $t_{1}$,

$$
n_{\mathrm{I}}=\zeta_{\mathrm{I}}=0 \text {, }
$$


et nous prendrons $\varepsilon=-\xi_{1}$, de telle façon que

$$
\xi_{\mathrm{I}}^{\prime}=n_{1}^{\prime}=\zeta_{\mathrm{I}}^{\prime}=0 \text {. }
$$

Nous pouvons donc ramener le calcul des deux ondes au cas où la vitesse de l'electron est nulle. Commençons par l'onde de vitesse; nous pouvons remarquer d'abord que cette onde est la même que si le mouvement de l'électron était uniforme.

Si la vitesse de l'électron est nulle, on a :

$$
\omega=0, \quad F=G=H=0, \quad \psi=\frac{\mu_{i}}{4 \pi r},
$$

$\mu_{\mathrm{r}}$ étant la charge électrique de l'électron. La vitesse ayant été ramenée à zéro par la transformation de LoRENTz, nous avons donc:

$$
F^{\prime}=G^{\prime}=H^{\prime}=0, \quad \psi^{\prime}=\frac{\mu_{1}}{4 \pi r^{\prime}},
$$

$r^{\prime}$ étant la distance du point $x^{\prime}, y^{\prime}, z^{\prime}$ au point $x_{1}^{\prime}, y_{1}^{\prime}, z_{1}^{\prime}$, et par conséquent:

$$
\begin{aligned}
\alpha^{\prime} & =\beta^{\prime}=\gamma^{\prime}=0, \\
f^{\prime}=\frac{\mu_{1}\left(x^{\prime}-x_{1}^{\prime}\right)}{4 \pi r^{\prime 3}}, \quad g^{\prime} & =\frac{\mu_{1}\left(y^{\prime}-y_{1}^{\prime}\right)}{4 \pi r^{\prime 3}}, \quad b^{\prime}=\frac{\mu_{1}\left(z^{\prime}-z_{1}^{\prime}\right)}{4 \pi r^{\prime 3}} .
\end{aligned}
$$

Faisons maintenant la transformation inverse de celle de LoRENTz pour trouver le champ véritable correspondant a une vitesse $-\varepsilon, 0$, o. Nous trouvons, en nous reportant aux équations (9) et (3) du $\ \mathrm{I}$ :

(4) $\left\{\begin{array}{c}\alpha=0, \quad \beta=\varepsilon h, \quad \gamma=-\varepsilon g, \\ f=\frac{\mu_{\mathrm{s}} k l^{3}}{4 \pi r^{\prime 3}}\left(x+\varepsilon t-x_{\mathrm{I}}-\varepsilon t_{\mathrm{s}}\right), \quad g=\frac{\mu_{\mathrm{s}} k l^{3}}{4 \pi r^{\prime 3}}\left(y-y_{\mathrm{s}}\right), \quad b=\frac{\mu_{\mathrm{s}} k l^{3}}{4 \pi r^{\prime 3}}\left(z-z_{\mathrm{s}}\right) .\end{array}\right.$

On voit que le champ magnétique est perpendiculaire à l'áxe des $x$ (direction de la vitesse) et au champ électrique, et que le champ électrique est dirigé vers le point:

$$
x_{1}+\varepsilon\left(t_{1}-t\right), \quad y_{1}, \quad z_{1} \text {. }
$$

Si l'électron continuait à se mouvoir d'un mouvement rectiligne et uniforme avec la vitesse qu'il avait à l'instant $t_{\imath}$, c'est-d-dire avec la vitesse $-\varepsilon, o$, o, ce point (s) serait celui qu'il occuperait d l'instant $t$.

Passons à l'onde d'accélération; nous pouvons, grâce à la transformation de LoRENTz, ramener sa détermination au cas où la vitesse est nulle. C'est le cas qui est réalisé si on imagine un électron qui exécute des oscillations d'amplitude très petites, mais très rapides, de façon que les déplacements et les vitesses soient infiniment petits, mais que les accélérations soient finies. On retombe ainsi sur le champ qui a été étudié dans le célèbre Mémoire de HerTz intitulé Die Kräfte elektrischer Schwingungen nach der Maxwell'schen Theorie et cela pour un point très éloigné. Dans ces conditions:

$\mathrm{I}^{\circ}$ Les deux champs électrique et magnétique sont égaux entre eux.

$2^{\circ}$ Ils sont perpendiculaires entre eux.

$3^{\circ}$ Ils sont perpendiculaires à la normale à la sphère d'onde, c'est-à-dire à la sphère dont le centre est le point $x_{1}, y_{2}, z_{1}$. 
H. POIN CAR É.

Je dis que ces trois propriétés subsisteront encore quand la vitesse ne sera pas nulle, et pour cela, il me suffit de montrer qu'elles ne sont pas altérćes par la transformation de LORENTz.

Soit en effet $A$ l'intensité commune des deux champs, soit

$$
\left(x-x_{1}\right)=r \lambda, \quad\left(y-y_{1}\right)=r \mu, \quad\left(z-z_{1}\right)=r \nu, \quad \lambda^{2}+\mu^{2}+\nu^{2}=\mathbf{I} .
$$

Ces propriétés s'exprimeront par les égalités:

$$
\begin{gathered}
A^{2}=\sum f^{2}=\sum \alpha^{2}, \quad \sum f \alpha=0, \quad \sum f\left(x-x_{1}\right)=0, \quad \sum \alpha\left(x-x_{1}\right)=0, \\
\sum f \lambda=0, \quad \sum \alpha \lambda=0 ;
\end{gathered}
$$

ce qui veut dire encore que

\begin{tabular}{ccc}
$\frac{b}{A}$, & $\frac{g}{A}$, & $\frac{b}{A}$ \\
$\alpha$ & $\beta$ & $\gamma$ \\
\hline$A$ & $\frac{\gamma}{A}$, & $\frac{\bar{A}}{2}$ \\
$\lambda$, & $\mu$, & $\nu$
\end{tabular}

sont les cosinus directeurs de trois directions rectangulaires, et on en déduit les relations :

ou

$$
f=\beta \nu-\gamma \mu, \quad \alpha=b \mu-g \nu,
$$

(6) $\quad f r=f\left(z-z_{\mathrm{I}}\right)-\gamma\left(y-y_{\mathrm{s}}\right), \quad \alpha r=b\left(y-y_{\mathrm{l}}\right)-g\left(z-z_{\mathrm{I}}\right)$,

avec les équations que l'on en peut déduire par symétrie.

Si nous reprenons les équations (3) $\mathrm{du} \int \mathrm{x}$, nous trouvons:

(7)

$$
\left\{\begin{array}{l}
x^{\prime}-x_{1}^{\prime}=k l\left[\left(x-x_{1}\right)+\varepsilon\left(t-t_{1}\right)\right]=k l\left[\left(x-x_{1}\right)+\varepsilon r\right], \\
y^{\prime}-y_{1}^{\prime}=l\left(y-y_{1}\right), \\
z^{\prime}-z_{1}^{\prime}=l\left(z-z_{1}\right) .
\end{array}\right.
$$

Nous avons trouvé plus haut au $\$ 3$ :

$$
l^{4}\left(\sum f^{2}-\sum \alpha^{\prime 2}\right)=\sum f^{2}-\sum \alpha^{2} .
$$

Donc $\sum f^{2}=\sum \alpha^{2}$ entraine $\sum f^{\prime 2}=\sum \alpha^{\prime 2}$.

D'autre part, en partant des équations (9) du $\ \mathrm{I}$, on trouve:

$$
l^{4} \sum f^{\prime} \alpha^{\prime}=\sum f \alpha,
$$

ce qui montre que $\sum f \alpha=0$ entraine $\sum f^{\prime} \alpha^{\prime}=0$.

Je dis maintenant que

$$
\sum f^{\prime}\left(x^{\prime}-x_{1}^{\prime}\right)=0, \quad \sum \alpha^{\prime}\left(x^{\prime}-x_{\mathrm{r}}^{\prime}\right)=0 .
$$

En effet, en vertu des équations (7) (ainsi que des équations 9 du $\$$ I) les premiers membres des deux équations ( 8$)$ s'écrivent respectivement:

$$
\begin{aligned}
& \frac{k}{l} \sum f\left(x-x_{1}\right)+\frac{k \Sigma}{l}\left[f r+\gamma\left(y-y_{\mathrm{s}}\right)-\beta\left(z-z_{1}\right)\right], \\
& \frac{k}{l} \sum \alpha\left(x-x_{1}\right)+\frac{k \varepsilon}{l}\left[\alpha r-b\left(y-y_{1}\right)+g\left(z-z_{1}\right)\right] .
\end{aligned}
$$


Ils s'annulent donc en vertu des équations $\sum f\left(x-x_{1}\right)=\sum x\left(x-x_{1}\right)=0$ et en vertu des équations (6). Or c'est là précisément ce qu'il s'agissait de démontrer.

On peut d'ailleurs arriver au même résultat par de simples considérations d'homogénéité.

En effet, $\psi, F, G, H$ sont des fonctions de $x-x_{1}, y-y_{1}, z-z_{1}, \xi_{1}=\frac{d x_{I}}{d t_{1}}$, $n_{1}=\frac{d y_{1}}{d t_{1}}, \zeta_{1}=\frac{d z_{1}}{d t_{1}}$ homogènes de degré - I par rapport d $x, y, z, t, x_{1}, y_{1}, z_{1}, t_{1}$ et à leurs différentielles.

Donc les dérivées de $\psi, F, G, H$ par rapport à $x, y$, z, $t$ (et par conséquent aussi les deux champs $f, g, b ; \alpha, \beta, \gamma)$ seront homogènes de degré -2 par rapport aux mêmes quantités, si nous nous rappelons d'ailleurs que la relation

est homogène par rapport à ces quantités.

$$
t-t_{1}=r=\sqrt{\sum\left(x-x_{1}\right)^{2}}
$$

Or ces dérivées ou ces champs dépendent des $x-x_{1}$, des vitesses $\frac{d x_{1}}{d t_{1}}$ et des accélérations $\frac{d^{2} x_{1}}{d t_{1}^{2}}$; ils se composent d'un terme indépendant des accélérations (onde de vitesse) et d'un terme linéaire par rapport aux accélérations (onde d'accélération). Or $\frac{d x_{1}}{d t_{1}}$ est homogène de degré o et $\frac{d^{2} x_{1}}{d t_{1}^{2}}$ homogène de degré - I; d'où il suit que l'onde de vitesse est homogène de degré -2 par rapport à $x-x_{1}, y-y_{1}, z-z_{1}$ et l'onde d'accélération homogène de degré - I. Donc, en un point très éloigné l'onde d'accélération est prépondérante et peut par conséquent être regardée comme se confondant avec l'onde totale. De plus, la loi d'homogénéité nous montre que l'onde d'accélération est semblable d elle-même en un point éloigné et en un point quelconque. Elle est donc, en un point quelconque, semblable à l'onde totale en un point éloigné. Or en un point éloigné la perturbation ne peut se propager que par ondes planes, de sorte que les deux champs doivent être égaux, perpendiculaires entre eux et perpendiculaires à la direction de propagation.

Je me bornerai à renvoyer pour plus de détails au Mémoire de M. Langevin dans le Journal de Physique (Année r9o5).

\section{6. - Contraction des Électrons.}

Supposons un électron unique animé d'un mouvement de translation rectiligne et uniforme. D'après ce que nous venons de voir, on peut, grâce d la transformation de LoRENTz, ramener l'étude du champ déterminé par cet électron au cas où l'électron serait immobile; la transformation de LoRENTz remplace donc l'électron réel en mouvement par un électron idéal immobile.

Soit $\alpha, \beta, \gamma ; f, g, b$ le champ réel; soit $\alpha^{\prime}, \beta^{\prime}, \gamma^{\prime} ; f^{\prime}, g^{\prime}, h^{\prime}$ ce que devient le 
champ après la transformation de LoRentz, de sorte que le champ idéal $\alpha^{\prime}, f^{\prime}$ correspond au cas d'un électron immobile; on a:

$$
\alpha^{\prime}=\xi^{\prime}=\gamma^{\prime}=0, \quad f^{\prime}=-\frac{d \psi^{\prime}}{d x^{\prime}}, \quad g^{\prime}=-\frac{d \psi^{\prime}}{d y^{\prime}}, \quad b^{\prime}=-\frac{d \psi^{\prime}}{d z^{\prime}} ;
$$

et pour le champ réel (en vertu des formules $9 \mathrm{du} \ \mathrm{I}$ ):

$$
\left\{\begin{array}{lll}
\alpha=0, & \beta=\varepsilon b, & \gamma=-\varepsilon g, \\
f=l^{2} f^{\prime}, & g=k l^{2} g^{\prime}, & b=k l^{2} b^{\prime} .
\end{array}\right.
$$

Il s'agit maintenant de déterminer l'énergie totale due au mouvement de l'électron, l'action correspondante et la quantité de mouvement électromagnétique, afin de pouvoir calculer les masses électromagnétiques de l'électron. Pour un point éloigné, il suffit de considérer l'électron comme réduit à un point unique; on est ainsi ramené aux formules (4) du $\$ précédent qui généralement peuvent convenir. Mais ici elles ne sauraient suffire, parce que l'energie est principalement localisée dans les parties de l'etther les plus voisines de l'électron.

On peut faire à ce sujet plusieurs hypothèses.

D'après celle d'Abraham, les électrons seraient sphériques et indéformables.

Alors, quand on appliquerait la transformation de LORENTz, comme l'électron réel serait sphérique, l'électron idéal deviendrait un ellipsoïde. L'équation de cet ellipsoïde serait d'après le $\ \mathrm{I}$ :

Mais ici l'on a:

$$
k^{2}\left(x^{\prime}-\varepsilon t^{\prime}-\xi t^{\prime}+\varepsilon \xi x^{\prime}\right)^{2}+\left(y^{\prime}-n k t^{\prime}+n k \varepsilon x^{\prime}\right)^{2}
$$

$$
+\left(z^{\prime}-\zeta k t^{\prime}+\zeta k \varepsilon x^{\prime}\right)^{2}=l^{2} r^{2} \text {. }
$$

$$
\xi+\varepsilon=n=\zeta=0, \quad \mathrm{I}+\varepsilon \xi=\mathrm{I}-\varepsilon^{2}=\frac{\mathrm{I}}{k^{2}},
$$

de sorte que l'équation de l'ellipsoïde devient:

$$
\frac{x^{\prime 2}}{k^{2}}+y^{\prime 2}+z^{\prime 2}=l^{2} r^{2} \text {. }
$$

Si le rayon de l'électron réel est $r$, les axes de l'électron idéal seraient donc:

$$
k l r, \quad l r, \quad l r .
$$

Dans l'hypothèse de LoRentz, au contraire, les électrons en mouvement seraient déformés, de telle façon que ce serait l'électron réel qui deviendrait un ellipsoïde, tandis que l'électron idéal immobile serait toujours une sphère de rayon $r$; les axes de l'électron réel seront alors:

Désignons par

$$
\frac{r}{l k}, \quad \frac{r}{l}, \quad \frac{r}{l} \text {. }
$$

$$
A=\frac{\mathrm{I}}{2} \int f^{2} d \tau
$$

l'énergie électrique longitudinale; par

$$
B=\frac{\mathrm{I}}{2} \int\left(g^{2}+b^{2}\right) d \tau
$$


l'énergie electrique transversale; par

$$
C=\frac{I}{2} \int\left(\beta^{2}+\gamma^{2}\right) d \tau
$$

l'énergie magnétique transversale. Il n'y a pas d'énergie magnétique longitudinale, puisque $\alpha=x^{\prime}=0$. Désignons par $A^{\prime}, B^{\prime}, C^{\prime}$ les quantités correspondantes dans le système idéal. On trouve d'abord:

$$
C^{\prime}=0, \quad C=\varepsilon^{2} B .
$$

D'autre part, nous pouvons observer que le champ réel dépend seulement de $x+\varepsilon t$, $y$ et $z$, et écrire :

d'où

$$
\begin{aligned}
& d \tau=d(x+\varepsilon t) d y d z, \\
& d \tau^{\prime}=d x^{\prime} d y^{\prime} d z^{\prime}=k l^{3} d \tau ;
\end{aligned}
$$

$$
A^{\prime}=k l^{-1} A, \quad B^{\prime}=k^{-1} l^{-1} B, \quad A=\frac{l A^{\prime}}{k}, \quad B=k l B^{\prime} .
$$

Dans l'hypothèse de Lorentz on a $B^{\prime}=2 A^{\prime}$, et $A^{\prime}$, inversement proportionnel au rayon de l'électron, est une constante indépendante de la vitesse de l'électron réel; on trouve ainsi pour l'energie totale:

$$
A+B+C=A^{\prime} l k\left(3+\varepsilon^{2}\right)
$$

et pour l'action (par unité de temps):

$$
A+B-C=\frac{3 A^{\prime} l}{k} \text {. }
$$

Calculons maintenant la quantité de mouvement électromagnétique; nous trouverons:

$$
D=\int(g \gamma-b \beta) d \tau=-\varepsilon \int\left(g^{2}+b^{2}\right) d \tau=-2 \varepsilon B=-4 \varepsilon k l A^{\prime} .
$$

Mais on doit avoir certaines relations entre l'énergie $E=A+B+C$, l'action par unité de temps $H=A+B-C$, et la quantité de mouvement $D$. La première de ces relations est:

la seconde est

$$
E=H-\varepsilon \frac{d H}{d \varepsilon}
$$

d'où :

$$
\frac{d D}{d \varepsilon}=-\frac{\mathrm{I}}{\varepsilon} \frac{d E}{d \varepsilon}
$$

$$
D=\frac{d H}{d \varepsilon}, \quad E=H-\varepsilon D .
$$

La seconde des équations (2) est toujours satisfaite; mais la première ne l'est que si

$$
l=\left(\mathbf{I}-\varepsilon^{2}\right)^{\frac{1}{6}}=k^{-\frac{x}{3}},
$$

c'est-à-dire si le volume de l'électron idéal est égal à celui de l'électron réel, ou encore si le volume de l'electron est constant; c'est l'hypothèse de Langevin. 
Cela est en contradiction avec le résultat du $\ 4$ et avec le résultat obtenu par LORENTz par une autre voie. C'est cette contradiction qu'il s'agit d'expliquer.

Avant d'aborder cette explication, j'observe que, quelle que soit l'hypothèse adoptée nous aurons

ou, à cause de $C^{\prime}=0$,

$$
H=A+B-C=\frac{l}{k}\left(A^{\prime}+B^{\prime}\right),
$$

(3)

$$
H=\frac{l}{k} H^{\prime} \text {. }
$$

Nous pouvons rapprocher ce résultat de l'équation $J=J^{\prime}$ obtenue au $\ 3$.

Nous avons en effet:

$$
J=\int H d t, \quad J^{\prime}=\int H^{\prime} d t^{\prime} .
$$

Nous observerons que l'état du système dépend seulement de $x+\varepsilon t, y$ et $z$, c'est-ḋ-dire de $x^{\prime}, y^{\prime}, z^{\prime}$, et que nous avons:

$$
\begin{aligned}
t^{\prime} & =\frac{l}{k} t+\varepsilon x^{\prime}, \\
d t^{\prime} & =\frac{l}{k} d t .
\end{aligned}
$$

En rapprochant les équations (3) et (4) on trouve $J=J$ '.

Plaçons-nous dans une hypothèse quelconque, qui pourra être, soit celle de LoRENTZ soit celle d'ABRAHAM, soit celle de LANGEvin, soit une hypothèse intermédiaire.

Soient

$$
r, \quad \theta r, \quad \theta r
$$

les trois axes de l'électron réel; ceux de l'électron idéal seront:

$$
\text { klr, } \quad \theta l . r, \quad \theta l r .
$$

Alors $A^{\prime}+B^{\prime}$ sera l'energie électrostatique due à un ellipsoïde ayant pour axes $k l r, \theta l r, \theta l r$.

Que l'on suppose l'électricité répandue d la surface de l'electron comme à celle d'un conducteur, ou uniformément répandue à l'intérieur de cet électron; cette énergie sera de la forme:

$$
A^{\prime}+B^{\prime}=\frac{\varphi\left(\frac{\theta}{k}\right)}{k l r},
$$

où $\varphi$ est une fonction connue.

L'hypothèse d'Abraham consiste d̀ supposer:

Celle de LOREnTz:

$$
r=\text { const. }, \quad \theta=\mathrm{I} \text {. }
$$

$$
l=\mathrm{I}, \quad k r=\text { const. }, \quad \theta=k \text {. }
$$


Celle de Langevin:

On trouve ensuite:

$$
l=k^{-\frac{s}{3}}, \quad k=\theta, \quad k l r=\text { const. }
$$

$$
H=\frac{\varphi\left(\frac{\theta}{k}\right)}{k^{2} r} .
$$

Aвraham trouve, à la différence des notations près (Göttinger Nachrichten, I902, p. 37):

$$
H=\frac{a}{r} \frac{\mathrm{I}-\varepsilon^{2}}{\varepsilon} \log \frac{\mathbf{I}+\varepsilon}{\mathrm{I}-\varepsilon},
$$

$a$ étant une constante. Or, dans l'hypothèse d'Abraham, on a $\theta=\mathrm{I}$; donc:

$$
\varphi\left(\frac{\mathrm{I}}{k}\right)=a k^{2} \frac{\mathrm{I}-\varepsilon^{2}}{\varepsilon} \log \frac{\mathrm{I}+\varepsilon}{\mathrm{I}-\varepsilon}=\frac{a}{\varepsilon} \log \frac{\mathrm{I}+\varepsilon}{\mathrm{x}-\varepsilon},
$$

ce qui définit la fonction $\varphi$.

Cela posé, imaginons que l'électron soit soumis à une liaison, de telle façon qu'il $\mathrm{y}$ ait une relation entre $r$ et $\theta$; dans l'hypothèse de LORENTz cette relation serait $\theta r=$ const., dans celle de Langevin $\theta^{2} r^{3}=$ const. Nous supposerons d'une façon plus générale

$b$ étant une constante; d'où :

$$
\begin{gathered}
r=b \theta^{m}, \\
H=\frac{I}{b k^{2}} \theta^{-m} \varphi\left(\frac{\theta}{k}\right) .
\end{gathered}
$$

Quelle sera la forme que prendra l'electron quand la vitesse deviendra $-\varepsilon t$, si l'on ne suppose pas l'intervention d'autres forces que celles de liaison? Cette forme sera définie par l'égalité:

$$
\frac{\partial H}{\partial \theta}=\mathrm{o}
$$

ou

ou

$$
-m \theta^{-m-1} \varphi+\theta^{-m} k^{-\mathrm{r}} \varphi^{\prime}=0,
$$

$$
\frac{\varphi^{\prime}}{\varphi}=\frac{m k}{\theta} \text {. }
$$

Si nous voulons que l'équilibre ait lieu de telle façon que $\theta=k$, il faut que pour $\frac{\theta}{k}=\mathrm{I}$, la dérivée logarithmique de $q$ soit égale à $m$.

Si nous développons $\frac{\mathrm{I}}{k}$ et le $2^{\mathrm{d}}$ membre de $(5)$ suivant les puissances de $\varepsilon$, l'equation $(5)$ devient:

$$
\varphi\left(\mathrm{I}-\frac{\varepsilon^{2}}{2}\right)=a\left(\mathrm{I}+\frac{\varepsilon^{2}}{3}\right)
$$

en négligeant les puissances supérieures de $\varepsilon$. 
En différentiant, il vient:

$$
-\varepsilon \varphi^{\prime}\left(\mathrm{I}-\frac{\varepsilon^{2}}{2}\right)=\frac{2}{3} \varepsilon a .
$$

Pour $\varepsilon=0$, c'est-d̀-dire quand l'argument de $\varphi$ est égal à $\mathrm{I}$, ces équations deviennent:

$$
\varphi=a, \quad \varphi^{\prime}=-\frac{2}{3} a, \quad \frac{\varphi^{\prime}}{\varphi}=-\frac{2}{3} .
$$

On doit donc avoir $m=-\frac{2}{3}$ conformément à l'hypothèse de Langevin.

Ce résultat doit être rapproché de celui qui est relatif à la I Ire équation (2) et dont en réalité il ne diffère pas. En effet, supposons que tout élément $d \tau$ de l'électron soit soumis d̀ une force $X d \tau$ parallèle d̀ l'axe des $x, X$ étant le même pour tous les éléments; nous aurons alors, conformément à la définition de la quantité de mouvement:

$$
\frac{d D}{d t}=\int X d \tau
$$

D'autre part, le principe de moindre action nous donne:

$$
\delta J=\int X \delta U d \tau d t, \quad J=\int H d t, \quad \delta J=\int D \delta U d t,
$$

$\delta U$ étant le déplacement du centre de gravité de l'électron; $H$ dépend de $\theta$ et de $\varepsilon$, si l'on admet que $r$ est lié d $\theta$ par l'équation de liaison; on a alors:

$$
\delta J=\int\left(\frac{\partial H}{\partial \varepsilon} \delta \varepsilon+\frac{\partial H}{\partial \theta} \delta \theta\right) d t
$$

D'autre part $\delta \varepsilon=-\frac{d \delta U}{d t}$; d'où, en intégrant par parties:

$$
\int D \delta \varepsilon d t=\int D \delta U d t
$$

ou

d'où

$$
\int\left(\frac{\partial H}{\partial \varepsilon} \delta \varepsilon+\frac{\partial H}{\partial \theta} \delta \theta\right) d t=\int D \delta \varepsilon d t
$$

$$
D=\frac{\partial H}{\partial \varepsilon}, \quad \frac{\partial H}{\partial \theta}=0 .
$$

Mais la dérivée $\frac{d H}{d \varepsilon}$, qui figure dans le $2^{\mathrm{d}}$ membre de la $I^{\text {ère }}$ équation (2), c'est la dérivée prise en supposant $\theta$ exprimé en fonction de $\varepsilon$, de sorte que

$$
\frac{d H}{d \varepsilon}=\frac{\partial H}{\partial \varepsilon}+\frac{\partial H}{\partial \theta} \frac{d \theta}{d \varepsilon} .
$$

L'équation (2) équivaut donc à l'équation (6).

La conclusion est que si l'électron est soumis d̀ une liaison entre ses trois axes, 
et si aucune autre force n'intervient en debors des forces de liaison, la forme que prendra cet électron, quand il sera animé d'une vitesse uniforme, ne pourra être telle que l'électron idéal correspondant soit une sphère, que dans le cas où la liaison sera que le volume soit constant, conformément à l'hypothèse de Langevin.

Nous sommes amenés de la sorte à nous poser le problème suivant: quelles forces supplémentaires, autres que les forces de liaison, serait-il nécessaire de faire intervenir pour rendre compte de la loi de LoRENTz ou, plus généralement, de toute loi autre que celle de Langevin?

L'hypothèse la plus simple, et la première que nous devions examiner, c'est que ces forces supplémentaires dérivent d'un potentiel spécial dérivant des trois axes de l'ellipsoïde, et par conséquent de $\theta$ et de $r$; soit $F(\theta, r)$ ce potentiel; dans ce cas l'action aura pour expression:

$$
J=\int[H+F(\theta, r)] d t
$$

et les conditions d'équilibre s'écriront:

$$
\frac{d H}{d \theta}+\frac{d F}{d \theta}=0, \quad \frac{d H}{d r}+\frac{d F}{d r}=0 .
$$

Si nous supposons $r$ et $\theta$ liés par la liaison $r=b \theta^{m}$, nous pourrons regarder $r$ comme fonction de $\theta$, envisager $F$ comme ne dépendant que de $\theta$ et conserver seulement la $\mathrm{I}^{\text {ere }}$ équation (8) avec:

$$
H=\frac{\varphi}{b k^{2} \theta^{m}}, \quad \frac{d H}{d \theta}=\frac{-m \varphi}{b k^{2} \theta^{m+1}}+\frac{\varphi^{\prime}}{b k^{3} \theta^{m}} .
$$

Il faut que, pour $k=\theta$, l'équation ( 8 ) soit satisfaite; ce qui donne, en tenant compte des équations (7):

d'oủ :

$$
\frac{d F}{d \theta}=\frac{m a}{b \theta^{m+3}}+\frac{2}{3} \frac{a}{b \theta^{m+3}},
$$

$$
F=\frac{-a}{b \theta^{m+2}} \frac{m+\frac{2}{3}}{m+2}
$$

et dans l'hypothèse de Lorentz, où $m=-\mathrm{I}$ :

$$
F=\frac{a}{3 b \theta} \text {. }
$$

Supposons maintenant qu'il n'y ait aucune liaison et, considérant $r$ et $\theta$ comme deux variables indépendantes, conservons les deux équations (8); il viendra:

$$
H=\frac{\varphi}{k^{2} r}, \quad \frac{d H}{d \theta}=\frac{q^{\prime}}{k^{3} r}, \quad \frac{d H}{d r}=\frac{-\varphi}{k^{2} r^{2}} .
$$

Les équations (8) doivent être satisfaites pour $k=\theta, r=b \theta^{m}$; ce qui donne:

$$
\frac{d F}{d r}=\frac{a}{b^{2} \theta^{2 m+2}}, \quad \frac{d F}{d \theta}=\frac{2}{3} \frac{a}{b \theta^{m+3}} .
$$


- Une des manières de satisfaire d̀ ces conditions est de poser:

$$
F=A r^{\alpha} \theta^{\beta}
$$

$A$, \% et $\beta$ étant des constantes; les équations (9) doivent être satisfaites pour $k=\theta$, $r=b 4^{m}$, ce qui donne:

$$
A \alpha b^{\alpha-1} \theta^{m \alpha-m+\xi}=\frac{a}{b^{2}()^{2 m+2}}, \quad A \nLeftarrow b^{\alpha} \theta^{m \alpha+\beta-1}=\frac{2}{3} \frac{a}{b{\theta^{m+3}}^{m}} .
$$

En identifiant on trouve

$$
\alpha=3 \gamma, \quad \xi=2 \gamma, \quad \gamma=-\frac{m+2}{3 m+2}, \quad A=\frac{a}{\alpha b^{\alpha+1}} .
$$

Mais le volume de l'ellipsoide est proportionnel $d r^{3} \theta^{2}$, de sorte que le potentiel supplémentaire est proportionnel à la puissance $\gamma$ du volume de l'électron.

Dans l'hypothese de LOREnTz, on a $m=-\mathrm{r}, \gamma=\mathrm{I}$.

On retrouve donc l'bypothèse de LORENTz à la condition d'ajouter un potentiel supplémentaire proportionnel au volume de l'electron.

L'hypothèse de LANGEVIN correspond à $\gamma=\infty$.

\section{\7. - Mouvement quasi-stationnaire.}

Il reste d voir si cette hypothèse sur la contraction des électrons rend compte de l'impossibilité de mettre en évidence le mouvement absolu, et je commencerai par étudier le mouvement quasi-stationnaire d'un électron isolé, ou soumis seulement à l'action d'autres électrons éloignés.

On sait qu'on appelle mouvement quasi-stationnaire un mouvement où les variations de la vitesse sont assez lentes pour que les énergies magnétique et électrique dues au mouvement de l'electron diffèrent peu de ce qu'elles seraient dans le mouvement uniforme; on sait également que c'est en partant de cette notion du mouvement quasistationnaire qu'Aвraham est arrivé à celle des masses électromagnétiques transversale et longitudinale.

Je crois devoir préciser. Soit $H$ notre action par unité de temps :

$$
H=\frac{\mathrm{I}}{2} \int\left(\sum f^{2}-\sum \alpha^{2}\right) d \tau,
$$

où nous ne considérons pour le moment que les champs électrique et magnétique dus au mouvement d'un électron isolé. Au $\$ précédent, considérant le mouvement comme uniforme, nous regardions $H$ comme dépendant de la vitesse $\xi, n, \zeta$ du centre de gravité de l'électron (ces trois composantes, dans le $₫$ précédent, avaient pour valeurs $-\varepsilon, 0,0)$ et des paramètres $r$ et $\theta$ qui définissent la forme de l'électron.

Mais si le mouvement n'est plus uniforme, $H$ dépendra non seulement des valeurs de $\xi, n, \zeta, r, \theta$ a l'instant considéré, mais des valeurs de ces mêmes quantités à d'autres instants qui pourront en différer de quantités de même ordre que le temps mis 
par la lumière pour aller d'un point à l'autre de l'electron; en d'autres termes, $H$ dépendra non seulement de $\xi, n, \zeta, r, \theta$, mais de leurs dérivées de tous les ordres par rapport au temps.

Eh bien, le mouvement sera dit quasi-stationnaire quand les dérivées partielles de $H$ par rapport aux dérivées successives de $\xi, n, \zeta, r, \theta$ seront négligeables devant les dérivées partielles de $H$ par rapport aux quantités $\xi, n, \zeta, r, \theta$ elles-mêmes.

Les équations d'un pareil mouvement pourront s'écrire:

(I) $\left\{\begin{aligned} \frac{d H}{d \theta}+\frac{d F}{d \theta} & =\frac{d H}{d r}+\frac{d F}{d r}=0, \\ \frac{d}{d t} \frac{d H}{d \xi}=-\int X d \tau, \quad \frac{d}{d t} \frac{d H}{d n} & =-\int Y d \tau, \quad \frac{d}{d t} \frac{d H}{d \zeta}=-\int Z d \tau .\end{aligned}\right.$

Dans ces équations, $F$ a la même signification que dans le $\$ précédent; $X, Y, Z$ sont les composantes de la force qui agit sur l'électron: cette force étant due uniquement aux champs électrique et magnétique produits par les autres électrons.

Observons que $H$ ne dépend de $\xi, n, \zeta$ que par l'intermédiaire de la combinaison

$$
V=\sqrt{\xi^{2}+n^{2}+\zeta^{2}}
$$

c'est-à-dire de la grandeur de la vitesse; on a donc, en appelant encore $D$ la quantité de mouvement:

$$
\frac{d H}{d \xi}=\frac{d H}{d V} \frac{\xi}{V}=-D \frac{\xi}{V}
$$

d'où :

avec

$$
\begin{aligned}
& -\frac{d}{d t} \frac{d H}{d \xi}=\frac{D}{V} \frac{d \xi}{d t}-D \frac{\xi}{V^{2}} \frac{d V}{d t}+\frac{d D}{d V} \frac{\xi}{V} \frac{d V}{d t}, \\
& -\frac{d}{d t} \frac{d H}{d n}=\frac{D}{V} \frac{d n}{d t}-D \frac{n}{V^{2}} \frac{d V}{d t}+\frac{d D}{d V} \frac{\eta}{V} \frac{d V}{d t},
\end{aligned}
$$

$$
V \frac{d V}{d t}=\sum \xi \frac{d \xi}{d t}
$$

Si nous prenons la direction actuelle de la vitesse pour axe des $x$, il vient:

$$
\xi=V, \quad n=\zeta=0, \quad \frac{d \xi}{d t}=\frac{d V}{d t} ;
$$

les équations (2) et $\left(2^{\text {bis }}\right)$ deviennent:

$$
-\frac{d}{d t} \frac{d H}{d \xi}=\frac{d D}{d V} \frac{d \xi}{d t}, \quad-\frac{d}{d t} \frac{d H}{d n}=\frac{D}{V} \frac{d n}{d t}
$$

et les trois dernières équations ( $\mathrm{r})$ :

$$
\frac{d D}{d V} \frac{d \xi}{d t}=\int X d \tau, \quad \frac{D}{V} \frac{d n}{d t}=\int Y d \tau, \quad \frac{D}{V} \frac{d \zeta}{d t}=\int Z d \tau .
$$


C'est pourquoi Abraham a donné à $\frac{d D}{d V}$ le nom de masse longitudinale et à $\frac{D}{V}$ le nom de masse transversale; rappelons que $D=\frac{d H}{d V}$.

Dans l'hypothèse de Lorentz, on a:

$$
D=-\frac{d H}{d V}=-\frac{\partial H}{\partial V}
$$

$\frac{\partial H}{\partial V}$ représentant la dérivée par rapport a $V$, après que $r$ et $\theta$ ont été remplacés par leurs valeurs en fonctions de $V$ tirées des deux premières équations (I); on aura d'ailleurs, après cette substitution,

$$
H=+A \sqrt{\mathrm{I}-V^{2}}
$$

Nous choisirons les unités de telle façon que le facteur constant $A$ soit égal a I, et je pose $\sqrt{\mathrm{I}-V^{2}}=b$, d'où :

$$
H=+b, \quad D=\frac{V}{b}, \quad \frac{d D}{d V}=b^{-3}, \quad \frac{d D}{d V} \frac{\mathrm{I}}{V^{2}}-\frac{D}{V^{3}}=b^{-3} .
$$

Nous poserons encore:

$$
M=V \frac{d V}{d t}=\sum \xi \frac{d \xi}{d t}, \quad X_{1}=\int X d \tau
$$

et nous trouverons pour l'équation du mouvement quasi-stationnaire :

$$
b^{-1} \frac{d \xi}{d t}+b^{-3} \xi M=X_{x} \text {. }
$$

Voyons ce que deviennent ces équations par la transformation de LoRENTz. Nous poserons: I $+\xi \varepsilon=\mu$, et nous aurons d'abord:

$$
\mu \xi^{\prime}=\xi+\varepsilon, \quad \mu n^{\prime}=\frac{n}{k}, \quad \mu \zeta^{\prime}=\frac{\zeta}{k},
$$

d'où l'on tire aisement

Nous avons également

$$
\mu b^{\prime}=\frac{b}{k} .
$$

d'où :

$$
d t^{\prime}=k \mu d t
$$

$$
\frac{d \xi^{\prime}}{d t^{\prime}}=\frac{d \xi}{d t} \frac{\mathrm{I}}{k^{3} \mu^{3}}, \frac{d \eta^{\prime}}{d t^{\prime}}=\frac{d n}{d t} \frac{\mathrm{I}}{k^{2} \mu^{2}}-\frac{d \xi}{d t} \frac{n \varepsilon}{k^{2} \mu^{3}}, \frac{d \zeta^{\prime}}{d t^{\prime}}=\frac{d \zeta}{d t} \frac{\mathrm{I}}{k^{2} \mu^{2}}-\frac{d \xi}{d t} \frac{\zeta \varepsilon}{k^{2} \mu^{3}},
$$

d'où encore:

et

$$
M^{\prime}=\frac{d \xi}{d t} \frac{\varepsilon b^{2}}{k^{3} \mu^{4}}+\frac{M}{k^{3} \mu^{3}}
$$

$$
b^{\prime-1} \frac{d \xi^{\prime}}{d t^{\prime}}+b^{\prime-3} \xi^{\prime} M^{\prime}=\left[b^{-1} \frac{d \xi}{d t}+b^{-3}(\xi+\varepsilon) M\right] \mu^{-1},
$$


(7)

$$
b^{-1} \frac{d n^{\prime}}{d t^{\prime}}+b^{-3} n^{\prime} M^{\prime}=\left(b^{-1} \frac{d n}{d t}+b^{-3} n M\right) \mu^{-1} b^{-1} .
$$

Reportons-nous maintenant aux équations ( $\mathrm{I}^{\mathrm{bis}}$ ) $\mathrm{du} \uparrow \mathrm{I}$; on peut y regarder $X_{\mathrm{r}}, Y_{\mathrm{r}}, Z_{\mathrm{r}}$ comme ayant la même signification que dans les équations (5). D'autre part, nous avons $l=1$ et $\frac{\rho^{\prime}}{\rho}=k u$; ces équations deviennent donc:

$$
\left\{\begin{array}{l}
X_{\mathrm{r}}^{\prime}=\mu^{-1}\left(X_{\mathrm{s}}+\varepsilon \sum X_{\mathrm{s}} \xi\right), \\
Y_{\mathrm{r}}^{\prime}=k^{-1} \mu^{-1} Y_{1} .
\end{array}\right.
$$

Calculons $\sum X_{\mathrm{s}} \xi$ à l'aide des équations (5), nous trouverons:

$$
\sum X_{\mathrm{r}} \xi=b^{-3} M
$$

d'où :

$$
\left\{\begin{array}{l}
X_{1}^{\prime}=\mu^{-1}\left(X_{1}+\varepsilon b^{-3} M\right) \\
Y_{1}^{\prime}=k^{-1} \mu^{-1} Y_{1}
\end{array}\right.
$$

En comparant les équations $(5),(6),(7)$ et (9), on trouve enfin:

$$
\left\{\begin{aligned}
b^{\prime-1} \frac{d \xi^{\prime}}{d t^{\prime}}+b^{\prime-3} \xi^{\prime} M^{\prime} & =X_{1}^{\prime}, \\
b^{\prime-1} \frac{d \eta^{\prime}}{d t^{\prime}}+b^{\prime-3} \eta^{\prime} M^{\prime} & =Y_{1}^{\prime},
\end{aligned}\right.
$$

ce qui montre que les équations du mouvement quasi-stationnaire ne sont pas altérées par la transformation de LORENTz; mais cela ne prouve pas encore que l'hypothèse de LORENTz est la seule qui conduise à ce résultat.

Pour établir ce point, nous allons nous restreindre, ainsi que l'a fait Lorentz, à certains cas particuliers, ce qui nous suffira évidemment pour démontrer une proposition négative.

Comment allons-nous d'abord étendre les hypothèses sur lesquelles reposait le calcul précédent?

$\mathrm{I}^{\circ}$ Au lieu de supposer $l=\mathrm{I}$ dans la transformation de LoRENTz, nous supposerons $l$ quelconque.

$2^{\circ} \mathrm{Au}$ lieu de supposer que $F$ est proportionnel au volume, et par conséquent que $H$ est proportionnel à $h$, nous supposerons que $F$ est une fonction quelconque de $\theta$ et de $r$, de telle façon que [après avoir remplacé $\theta$ et $r$ par leurs valeurs en fonctions de $V$, tirées des deux premières équations (I)] $H$ soit une fonction quelconque de $V$.

J'observe d'abord que, si l'on suppose $H=b$, on devra avoir $l=\mathrm{I}$; et en effet les équations (6) et (7) subsisteront, sauf que les seconds membres seront multipliés par $\frac{I}{l}$; les équations (9) également, sauf que les seconds membres seront multipliés par $\frac{\mathrm{I}}{l^{2}}$; et enfin les équations ( $\mathrm{I} 0$ ), sauf que les seconds membres seront multipliés par $\frac{\mathrm{I}}{l} \cdot \mathrm{Si}$ l'on veut que les équations du mouvement ne soient pas alterées par la transformation 
de LoRentz, c'est-à-dire que les équations (IO) ne diffèrent des équations ( 5 ) que par l'accentuation des lettres, il faut supposer:

$$
l=\mathrm{r} .
$$

Supposons maintenant que l'on ait $n=\zeta=0$, d'où $\xi=V, \frac{d \xi}{d t}=\frac{d V}{d t}$; les équations ( $s$ ) prendront la forme:

$\left(\varsigma^{\text {bis }}\right) \quad-\frac{d}{d t} \frac{d H}{d \xi}=\frac{d D}{d V} \frac{d \xi}{d t}=X_{i}, \quad-\frac{d}{d t} \frac{d H}{d n}=\frac{D}{V} \frac{d n}{d t}=Y_{\mathrm{i}}$.

Nous pouvons d'ailleurs poser:

$$
\frac{d}{d} \frac{D}{V}=f(V)=f(\xi), \quad \frac{D}{V}=\varphi(V)=\varphi(\xi) .
$$

Si les équations du mouvement ne sont pas altérées par la transformation de LoRENTZ, on devra avoir:

$$
\begin{aligned}
& f(\xi) \frac{d \xi}{d t}=X_{1}, \\
& \varphi(\xi) \frac{d \eta}{d t}=Y_{1}, \\
& f\left(\xi^{\prime}\right) \frac{d \xi^{\prime}}{d t^{\prime}}=X_{1}^{\prime}=l^{-2} \mu^{-1}\left(X_{1}+\varepsilon \sum X_{1} \xi\right)=l^{-2} \mu^{-1} X_{1}(1+\varepsilon \xi)=l^{-2} X_{1}, \\
& \varphi\left(\xi^{\prime}\right) \frac{d \eta^{\prime}}{d t^{\prime}}=Y_{1}^{\prime}=l^{-2} k^{-1} \mu^{-1} Y_{1},
\end{aligned}
$$

et par conséquent :

$$
\left\{\begin{array}{l}
f(\xi) \frac{d \xi}{d t}=l^{2} f\left(\xi^{\prime}\right) \frac{d \xi^{\prime}}{d t^{\prime}}, \\
\varphi(\xi) \frac{d n_{1}}{d t}=l^{2} k \mu \varphi\left(\xi^{\prime}\right) \frac{d n^{\prime}}{d t^{\prime}} .
\end{array}\right.
$$

Mais nous avons:

d'où :

$$
\frac{d \xi^{\prime}}{d t^{\prime}}=\frac{d \xi}{d t} \frac{\mathrm{I}}{k^{3} \mu^{3}}, \quad \frac{d n^{\prime}}{d t^{\prime}}=\frac{d \eta}{d t} \frac{\mathrm{I}}{k^{2} \mu^{2}}
$$

$$
\begin{aligned}
& f\left(\xi^{\prime}\right)=f\left(\frac{\xi+\varepsilon}{I+\xi \varepsilon}\right)=f(\xi) \frac{k^{3} \mu^{3}}{l^{2}}, \\
& \varphi\left(\xi^{\prime}\right)=\varphi\left(\frac{\xi+\varepsilon}{I+\xi_{\varepsilon}}\right)=\varphi(\xi) \frac{k \mu}{l^{2}} ;
\end{aligned}
$$

d'où, en eliminant $l^{2}$, nous trouvons l'equation fonctionnelle:

$$
k^{2} \mu^{2} \frac{\varphi\left(\frac{\xi+\varepsilon}{I+\xi_{\varepsilon}}\right)}{\varphi(\xi)}=\frac{f\left(\frac{\xi+\varepsilon}{I+\xi_{\varepsilon}}\right)}{f(\xi)},
$$


ou, en posant

$$
\frac{\varphi(\xi)}{f(\zeta)}=\Omega(\xi)=\frac{D}{V \frac{d D}{d V}}
$$

celle-ci :

$$
\Omega\left(\frac{\xi+\varepsilon}{I+\xi \varepsilon}\right)=\Omega(\xi) \frac{I+t^{2}}{(I+\xi \varepsilon)^{2}},
$$

équation qui doit être satisfaite pour toutes les valeurs de $\xi$ et de $\varepsilon$. Pour $\zeta=0$ on trouve :

d'où :

$$
\Omega(\varepsilon)=\Omega(0)\left(\mathrm{I}-\varepsilon^{2}\right),
$$

$$
D=A\left(\frac{V}{\sqrt{\mathrm{I}-V^{2}}}\right)^{m}
$$

$A$ étant une constante, et où j'ai fait $\mathbf{\Omega}(\mathrm{o})=\frac{\mathrm{I}}{\mathrm{m}}$.

On trouve alors:

$$
\varphi(\xi)=\frac{A}{\xi}\left(\frac{\xi}{\sqrt{1-\xi^{2}}}\right)^{m}, \quad \varphi\left(\xi^{\prime}\right)=\frac{A \mu}{\xi+\varepsilon}\left(\frac{\xi+\varepsilon}{\sqrt{1-\xi^{2}} \frac{1}{\sqrt{\mathrm{I}-\varepsilon^{2}}}}\right)^{m} .
$$

Or $\varphi\left(\xi^{\prime}\right)=\varphi(\xi) \frac{k \mu}{l^{2}}$; donc on $\mathrm{a}$ :

$$
(\xi+\varepsilon)^{m-1}\left(1-\varepsilon^{2}\right)^{-\frac{m}{2}}=-\xi^{m-1}\left(1-\varepsilon^{2}\right)^{-\frac{1}{2}} l^{-2} .
$$

Comme $l$ ne doit dépendre que de $\varepsilon$ (puisque, s'il y a plusieurs électrons, $l$ doit être le même pour tous les électrons dont les vitesses $\zeta$ peuvent être différentes), cette identité ne peut avoir lieu que si l'on a:

$$
m=\mathrm{I}, \quad l=\mathrm{I} \text {. }
$$

Ainsi l'hypothèse de Lorentz est la seule qui soit compatible avec l'impossibilité de mettre en évidence le mouvement absolu; si on admet cette impossibilité, il faut admettre que les électrons en mouvement se contractent de façon à devenir des ellipsoïdes de révolution dont deux des axes demeurent constants; il faut donc admettre, comme nous l'avons montré au $\$ précédent, l'existence d'un potentiel supplémentaire proportionnel au volume de l'electron.

L'analyse de LoRENTz se trouve donc pleinement confirmée, mais nous pouvons mieux nous rendre compte de la vraie raison du fait qui nous occupe; cette raison doit être cherchée dans les considérations du $\ 4$. Les transformations qui n'altèrent pas les équations du mouvement doivent former un groupe, et cela ne peut avoir lieu que si $l=\mathrm{I}$. Comme nous ne devons pas pouvoir reconnaitre si un électron est en repos ou en mouvement absolu, il faut que quand il est en mouvement il subisse une déformation qui doit être précisément celle que lui impose la transformation correspondante du groupe. 


\section{§ 8. - Mouvement quelconque.}

Les résultats précédents ne s'appliquent qu'au mouvement quasi-stationnaire, mais il est aisé de les étendre au cas général; il suffit d'appliquer les principes du $\ 3$, c'estd-dire de partir du principe de moindre action.

A l'expression de l'action:

$$
J=\int d t d \tau\left(\frac{\sum f^{2}}{2}-\frac{\sum \alpha^{2}}{2}\right)
$$

il convient d'ajouter un terme, représentant le potentiel supplémentaire $F$ du $\ 6$; ce terme prendra évidemment la forme:

$$
J_{1}=\int \sum(F) d t
$$

où $\sum(F)$ représente la somme des potentiels supplémentaires dus aux différents ćlectrons, chacun d'eux étant proportionnel au volume de l'electron correspondant.

J'écris $(F)$ entre parenthèses pour ne pas confondre avec le vecteur $F, G, H$.

L'action totale est alors $J+J_{1}$. Nous avons vu au $\ 3$ que $J$ n'est pas altéré par la transformation de LoRENTz; il faut montrer maintenant qu'il en est de même de $J_{1}$.

On a, pour l'un des électrons,

$$
(F)=\omega_{0} \tau
$$

$\omega_{0}$ étant un coefficient spécial à l'électron et $\tau$ son volume; je puis donc écrire:

$$
\Sigma(F)=\int \omega_{0} d \tau
$$

l'intégrale devant être étendue d̀ tout l'espace, mais de telle façon que le coefficient $\omega_{0}$ soit nul en dehors des électrons, et qu'à l'intérieur de chaque électron il soit égal au coefficient spécial d̀ cet électron. On a alors:

$$
J_{\mathrm{s}}=\int \omega_{\mathrm{o}} d \tau d t
$$

et après la transformation de LORENTZ:

$$
J_{\mathrm{r}}^{\prime}=\int \omega_{0}^{\prime} d \tau^{\prime} d t^{\prime}
$$

Or on a $\omega_{0}=\omega_{o}^{\prime}$; car si un point appartient $\mathrm{a}$ un électron, le point correspondant après la transformation de LORENTZ appartient encore au même électron. D'autre part, nous avons trouvé au $₫ 3$ :

$$
d \tau^{\prime} d t^{\prime}=l^{4} d \tau d t
$$

et, puisque nous supposons maintenant $l=\mathrm{I}$,

$$
d \tau^{\prime} d t^{\prime}=d \tau d t
$$


On a donc

$$
J_{\mathrm{I}}=J_{\mathrm{i}}^{\prime} \text { C. Q. F. D. }
$$

Le théorème est donc général, il nous donne en même temps une solution de la question que nous nous posions à la fin du $\$ \mathbf{I}$ : trouver des forces complémentaires non altérées par la transformation de LoRentz. Le potentiel supplémentaire $(F)$ satifait à cette condition.

Nous pouvons donc généraliser le résultat énoncé à la fin du $\$ I et écrire:

Si l'inertie des électrons est exclusivement d'origine électromagnétique, s'ils ne sont soumis qu'à des forces d'origine dlectromagnétique, ou aux forces qui engendrent le potentiel supplémentaire $(F)$, aucune expérience ne pourra mettre en évidence le mouvement absolu.

Quelles sont alors ces forces qui engendrent le potentiel $(F)$ ? Elles peuvent évidemment être assimilees à une pression qui régnerait d l'intérieur de l'électron; tout se passe comme si chaque électron était une capacité creuse soumise à une pression interne constante (indépendante du volume); le travail d'une pareille pression serait évidemment proportionnel aux variations du volume.

Je dois observer toute fois que cette pression est négative. Reprenons l'equation (I0) du $\int 6$, qui dans l'hypothèse de LORENTz s'écrit:

$$
F=A r^{3} \theta^{2}
$$

les équations (II) du $₫ 6$ nous donneront:

$$
A=\frac{a}{3 b^{4}}
$$

Notre pression est égale à $A$, a un coefficient constant près, qui d'ailleurs est négatif.

Évaluons maintenant la masse de l'électron, je veux parler de la " masse expérimentale ", c'est-à-dire de la masse pour des vitesses faibles; on a (cf. $₫ 6$ ):

d'où

$$
H=\frac{\varphi\left(\frac{\theta}{k}\right)}{k^{2} r}, \quad \theta=k, \quad \varphi=a, \quad \theta r=b ;
$$

$$
H=\frac{a}{b k}=\frac{a}{b} \sqrt{\mathrm{I}-V^{2}} .
$$

Pour $V$ très petit je puis écrire:

$$
H=\frac{a}{b}\left(\mathrm{I}-\frac{V^{2}}{2}\right)
$$

de sorte que la masse, tant longitudinale que transversale, sera $\frac{a}{b}$.

Or $a$ est une constante numérique, ce qui montre que: la pression qui engendre notre potentiel supplémentaire est proportionnelle à la $4^{e}$ puissance de la masse expérimentale de l'electron. 
Comme l'attraction newtonienne est proportionnelle à cette masse expérimentale, on est tenté de conclure qu'il y a quelque relation entre la cause qui engendre la gravitation et celle qui engendre ce potentiel supplémentaire.

\section{9. - Hypothèses sur la Gravitation.}

Ainsi la théorie de Lorentz expliquerait complètement l'impossibilité de mettre en évidence le mouvement absolu, si toutes les forces étaient d'origine électromagnétique.

Mais il y a des forces auxquelles. on ne peut pas attribuer une origine électromagnétique comme par exemple la gravitation. Il peut arriver, en effet, que deux systèmes de corps produisent des champs électromagnétiques équivalents, c'est-d̀-dire exerçant la même action sur des corps électrisés et sur des courants, et que cependant ces deux systèmes n'exercent pas la même action gravifique sur les masses newtoniennes. Le champ gravifique est donc distinct du champ électromagnétique. LoRENTz a donc été obligé de compléter son hypothèse en supposant que les forces de toute origine, et en particulier la gravitation, sont affectées par une translation (ou, si l'on aime mieux, par la transformation de LORENTz) de la même manière que les forces électromagnétiques.

Il convient maintenant d'entrer dans les détails et d'examiner de plus près cette hypothèse. Si nous voulons que la force newtonienne soit affectée de cette façon par la transformation de LoRENTz, nous ne pouvons plus admettre que cette force dépend uniquement de la position relative du corps attirant et du corps attiré à l'instant considéré. Elle devra dépendre en outre des vitesses des deux corps. Et ce n'est pas tout: il sera naturel de supposer que la force qui agit à l'instant $t$ sur le corps attiré, dépend de la position et de la vitesse de ce corps à ce même instant $t$; mais elle dépendra, en outre, de la position et de la vitesse du corps attirant, non pas à l'instant $t$, mais à un instant antérieur, comme si la gravitation avait mis un certain temps à se propager.

Envisageons donc la position du corps attiré à l'instant $t_{0}$ et soient, à cet instant,

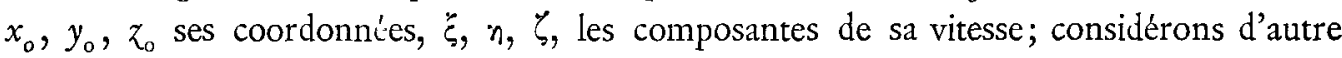
part le corps attirant a l'instant correspondant $t_{\mathrm{o}}+t$ et soient, a cet instant, $x_{\mathrm{o}}+x$, $y_{0}+y, z_{0}+z$ ses coordonnées, $\xi_{I}, n_{I}, \zeta_{I}$ les composantes de sa vitesse.

Nous devrons d'abord avoir une relation

$$
\varphi\left(t, x, y, z, \xi, n, \zeta, \xi_{1}, n_{1}, \zeta_{1}\right)=0
$$

pour définir le temps $t$. Cette relation définira la loi de la propagation de l'action gravifique (je ne m'impose nullement la condition que la propagation se fasse avec la même vitesse dans tous les sens).

Soient maintenant $X_{1}, Y_{1}, Z_{1}$ les 3 composantes de l'action exercée à l'instant $t$ sur le corps attiré; il s'agit d'exprimer $X_{1}, Y_{I}, Z_{I}$ en fonctions de

$$
t, x, y, z, \xi, n, \zeta, \xi_{1}, n_{1}, \zeta_{1} \text {. }
$$

Quelles sont les conditions à remplir? 
$I^{\circ}$ La condition (I) ne devra pas être altérée par les transformations du groupe de LoRentz.

$2^{\circ}$ Les composantes $X_{1}, Y_{1}, Z_{1}$ devront être affectées par les transformations de LoREntz de la même manière que les forces électromagnétiques désignées par les mêmes lettres, c'est-à-dire conformément aux équations (I ${ }^{\text {bis }}$ ) du $\ \mathrm{I}$.

$3^{\circ}$ Quand les deux corps seront au repos, on devra retomber sur la loi ordinaire de l'attraction.

Il importe de remarquer que dans ce dernier cas, la relation (I) disparait, car le temps $t$ ne joue plus aucun rôle si les deux corps sont au repos.

Le problème ainsi posé est évidemment indéterminé. Nous chercherons donc à satisfaire autant que possible d d'autres conditions complémentaires:

$4^{\circ}$ Les observations astronomiques ne semblant pas montrer de dérogation sensible à la loi de Newton, nous choisirons la solution qui s'écarte le moins de cette loi, pour de faibles vitesses des deux. corps.

$5^{\circ}$ Nous nous efforcerons de nous arranger de façon que $t$ soit toujours négatif; si en effet on conçoit que l'effet de la gravitation demande un certain temps pour se propager, il serait plus difficile de comprendre comment cet effet pourrait dépendre de la position non encore atteinte par le corps attirant.

Il y a un cas où l'indétermination du problème disparait; c'est celui où les deux corps sont en repos relatif l'un par rapport à l'autre, c'est-à-dire où :

$$
\xi=\xi_{1}, \quad n=n_{1}, \quad \zeta=\zeta_{1} ;
$$

c'est donc le cas que nous allons examiner d'abord, en supposant que ces vitesses sont constantes, de telle sorte que les deux corps sont entrainés dans un mouvement de translation commun, rectiligne et uniforme.

Nous pourrons supposer que l'axe des $x$ a été pris parallèle à cette translation, de telle façon que $n=\zeta=0$, et nous prendrons $\varepsilon=-\zeta$.

$\mathrm{Si}$ dans ces conditions nous appliquons la transformation de LoREnTz, après la transformation les deux corps seront au repos et l'on aura:

$$
\xi^{\prime}=n^{\prime}=\xi^{\prime}=0 \text {. }
$$

Alors les composantes $X_{\mathrm{I}}^{\prime}, Y_{\mathrm{t}}^{\prime}, Z_{\mathrm{t}}^{\prime}$ devront être conformes à la loi de Newton et on aura, à un facteur constant près :

$$
\left\{\begin{array}{c}
X_{\mathrm{r}}^{\prime}=-\frac{x^{\prime}}{r^{\prime 3}}, \quad Y_{\mathrm{r}}^{\prime}=-\frac{y^{\prime}}{r^{\prime 3}}, \quad Z_{\mathrm{r}}^{\prime}=-\frac{z^{\prime}}{r^{\prime 3}}, \\
r^{\prime 2}=x^{\prime 2}+y^{\prime 2}+z^{\prime 2} .
\end{array}\right.
$$

Mais on a, d'après le $\$ \mathrm{I}$ :

$$
\begin{aligned}
& x^{\prime}=k(x+\varepsilon t), \quad y^{\prime}=y, \quad z^{\prime}=z, \quad t^{\prime}=k(t+\varepsilon x), \\
& \frac{\rho^{\prime}}{\rho}=k(\mathrm{I}+\xi \varepsilon)=k\left(\mathrm{I}-\varepsilon^{2}\right)=\frac{\mathrm{I}}{k}, \quad \sum X_{1} \xi=-X_{\mathrm{s}} \varepsilon,
\end{aligned}
$$




$$
\begin{aligned}
& X_{\mathrm{r}}^{\prime}=k \frac{\rho}{\rho^{\prime}}\left(X_{\mathrm{r}}+\varepsilon \sum X_{\mathrm{r}} \xi\right)=k^{2} X_{\mathrm{r}}\left(\mathrm{I}-\varepsilon^{2}\right)=X_{\mathrm{r}}, \\
& Y_{\mathrm{r}}^{\prime}=\frac{\rho}{\rho^{\prime}} Y_{\mathrm{r}}=k Y_{\mathrm{r}}, \\
& Z_{\mathrm{r}}^{\prime}=k Z_{\mathrm{l}} .
\end{aligned}
$$

On a d'ailleurs:

et

$$
x+\varepsilon t=x-\xi t, \quad r^{\prime 2}=k^{2}(x-\xi)^{2}+y^{2}+z^{2}
$$

$$
X_{\mathrm{r}}=\frac{-k(x-\xi t)}{r^{\prime 3}}, \quad Y_{\mathrm{r}}=\frac{-y}{k r^{\prime 3}}, \quad Z_{\mathrm{r}}=\frac{-z}{k r^{\prime 3}} ;
$$

ce qui peut s'écrire:

$$
X_{\mathrm{r}}=\frac{d V}{d x}, \quad Y_{1}=\frac{d V}{d y}, \quad Z_{\mathrm{s}}=\frac{d V}{d z} ; \quad V=\frac{\mathrm{I}}{k r^{\prime}} .
$$

Il semble d'abord que l'indétermination subsiste, puisque nous n'avons fait aucune hypothèse sur la valeur de $t$, c'est-d-dire sur la rapidité de la transmission; et que d'ailleurs $x$ est fonction de $t$; mais il est aisé de voir que $x-\xi t, y, z$, qui figurent seuls dans nos formules, ne dépendent pas de $t$.

On voit que si les deux corps sont simplement animés d'une translation commune, la force qui agit sur le corps attiré est normale à un ellipsoide ayant pour centre le corps attirant.

Pour aller plus loin il faut chercher les invariants du groupe de LoRENTz.

Nous savons que les substitutions de ce groupe (en supposant $l=1$ ) sont les substitutions linéaires qui n'altèrent pas la forme quadratique

Posons d'autre part:

$$
x^{2}+y^{2}+z^{2}-t^{2} \text {. }
$$

$$
\begin{array}{lll}
\xi=\frac{\delta x}{\delta t}, & n=\frac{\delta y}{\delta t}, & \zeta=\frac{\delta z}{\delta t} ; \\
\xi_{\mathrm{r}}=\frac{\delta_{\mathrm{r}} x}{\delta_{\mathrm{I}} t}, & n_{\mathrm{I}}=\frac{\delta_{1} y}{\delta_{1} t}, & \zeta_{\mathrm{I}}=\frac{\delta_{\mathrm{I}} z}{\delta_{\mathrm{I}} t} ;
\end{array}
$$

nous voyons que la transformation de LORENTz aura pour effet de faire subir $₫ \delta x$, $\delta y, \delta z, \delta t$, et $\mathrm{a} \delta_{1} x, \delta_{1} y, \delta_{1} z, \delta_{1} t$ les mêmes substitutions linéaires qu'd $x, y, z, t$.

Regardons

$$
\begin{array}{cccc}
x, & y, & z, & t \sqrt{-\mathrm{I}}, \\
\delta x, & \delta y, & \delta z, & \delta t \sqrt{-\mathrm{I}}, \\
\delta_{\mathrm{I}} x, & \delta_{\mathrm{i}} y, & \delta_{\mathrm{I}} z, & \delta_{\mathrm{t}} t \sqrt{-\mathrm{I}},
\end{array}
$$

comme les coordonnées de 3 points $P, P^{\prime}, P^{\prime \prime}$ dans l'espace d̀ 4 dimensions. Nous voyons que la transformation de LORENTZ n'est qu'une rotation de cet espace autour de l'origine, regardée comme fixe. Nous n'aurons donc pas d'autres invariants distincts que les 6 distances des 3 points $P, P^{\prime}, P^{\prime \prime}$ entre eux et à l'origine, ou, si l'on aime 
mieux, que les 2 expressions:

$$
x^{2}+y^{2}+z^{2}-t^{2}, \quad x \delta x+y \delta y+z \delta z-t \delta t,
$$

ou les 4 expressions de même forme qu'on en déduit en permutant d'une manière quelconque les 3 points $P, P^{\prime}, P^{\prime \prime}$.

Mais ce que nous cherchons ce sont les fonctions des io variables (2) qui sont des invariants; nous devons donc, parmi les combinaisons de nos 6 invariants, rechercher celles qui ne dépendent que de ces Io variables, c'est-à-dire celles qui sont homogènes de degré o tant par rapport à $\delta x, \delta y, \delta z, \delta t$, que par rapport à $\delta_{\mathrm{x}} x, \delta_{1} y$, $\delta_{\mathrm{I}} z \delta_{\mathrm{I}} t$. Il nous restera ainsi 4 invariants distincts, qui sont:
(5) $\sum x^{2}-t^{2}$,
$\frac{t-\sum x \xi}{\sqrt{\mathrm{I}-\sum \xi^{2}}}$
$\frac{t-\sum x \xi_{1}}{\sqrt{1-\sum \xi_{I}^{2}}}$
$\frac{I-\sum \xi \xi_{I}}{\sqrt{\left(I-\sum \xi^{2}\right)\left(I-\sum \xi_{I}^{2}\right)}}$.

Occupons-nous maintenant des transformations subies par les composantes de la force; reprenons les équations (II) du $\oint_{\mathrm{I}}$, qui se rapportent non a la force $X_{1}, Y_{1}$, $Z_{1}$, que nous considérons ici, mais à la force $X, Y, Z$ rapportée à l'unité de volume. Posons d'ailleurs:

$$
T=\sum X \xi ;
$$

nous verrons que ces équations (II) peuvent s'ecrire $(l=\mathrm{I})$ :

$$
\begin{cases}X^{\prime}=k(X+\varepsilon T), & T^{\prime}=k(T+\varepsilon X), \\ Y^{\prime}=Y, & Z^{\prime}=Z\end{cases}
$$

de sorte que $X, Y, Z, T$ subissent la même transformation que $x, y, z, t$. Les invariants du groupe seront donc

$$
\sum X^{2}-T^{2}, \quad \sum X x-T t, \quad \sum X \delta x-T \delta t, \quad \sum X \delta_{1} x-T \delta_{1} t .
$$

Mais ce n'est pas de $X, Y, Z$ que nous avons besoin, c'est de $X_{1}, Y_{\mathrm{t}}, Z_{1}$, avec

Nous voyons que

$$
T_{\mathrm{r}}=\sum X_{\mathrm{r}} \xi \text {. }
$$

$$
\frac{X_{\mathrm{r}}}{X}=\frac{Y_{\mathrm{s}}}{Y}=\frac{Z_{\mathrm{s}}}{Z}=\frac{T_{\mathrm{s}}}{T}=\frac{\mathrm{r}}{\rho} \text {. }
$$

Done la transformation de LoRentz agira sur $X_{1}, Y_{1}, Z_{1}, T_{1}$ de la même manière que sur $X, Y, Z, T$, avec cette différence que ces expressions seront en outre multipliées par

$$
\frac{\rho}{\rho^{\prime}}=\frac{\mathrm{I}}{k\left(\mathrm{I}+\xi_{\varepsilon}\right)}=\frac{\delta t}{\delta t^{\prime}} \text {. }
$$

De même elle agira sur $\xi, n, \zeta, \mathrm{I}$, de la même manière que sur $\delta x, \delta y, \delta z \delta t$, avec cette différence que ces expressions seront en outre multipliées par le même facteur:

$$
\frac{\delta t}{\delta t^{\prime}}=\frac{\mathrm{I}}{k(\mathrm{I}+\xi \varepsilon)} \text {. }
$$

Considérons alors $X, Y, Z, T \sqrt{-1}$ comme les coordonnées d'un quatrième point 
$Q$; alors les invariants seront les fonctions des distances mutuelles des cinq points

$$
0, P, P^{\prime}, P^{\prime \prime}, Q
$$

et parmi ces fonctions nous devons conserver seulement celles qui sont homogènes de degré o, d'une part par rapport à

$$
X, \quad Y, Z, T, \delta x, \delta y, \delta z, \delta t
$$

(variables que l'on peut remplacer ensuite par $X_{1}, Y_{1}, Z_{1}, T_{1}, \xi, n, \zeta, \mathrm{I}$ ), d'autre part par rapport à

$$
\delta_{\mathrm{I}} x, \delta_{\mathrm{I}} y, \delta_{\mathrm{I}} z, \mathrm{I}
$$

(variables que l'on peut remplacer ensuite par $\xi_{1}, n_{1}, \zeta_{1}, I$ ).

Nous trouvons ainsi outre les quatre invariants $(5)$, quatre invariants distincts nouveaux, qui sont:

(7) $\frac{\sum X_{1}^{2}-T_{1}^{2}}{I-\sum \xi^{2}}, \frac{\sum X_{I} x-T_{1} t}{\sqrt{I-\sum \xi^{2}}}, \frac{\sum X_{1} \xi_{1}-T_{1}}{\sqrt{I-\sum \xi^{2}} \sqrt{I-\sum \xi_{I}^{2}}}, \frac{\sum X_{I} \xi-T_{I}}{I-\sum \xi^{2}}$.

Le dernier invariant est toujours nul, d'après la definition de $T_{s}$.

Cela posé, quelles sont les conditions à remplir?

$I^{\circ}$ Le premier membre de la relation (I), qui définit la vitesse de propagation, doit être une fonction des 4 invariants ( 5 ).

On peut faire évidemment une foule d'hypothèses; nous n'en examinerons que deux :

A) On peut avoir

$$
\sum x^{2}-t^{2}=r^{2}-t^{2}=0,
$$

d'où $t= \pm r$, et, puisque $t$ doit être négatif, $t=-r$. Cela veut dire que la vitesse de propagation est égale à celle de la lumière. Il semble d'abord que cette hypothèse doive être rejetée sans examen. LAPLACE a montré en effet que la propagation est, ou bien instantanée, ou beaucoup plus rapide que celle de la lumière. Mais LAPLACE avait examiné l'hypothèse de la vitesse finie de propagation, ceteris non mutatis; ici, au contraire, cette hypothèse est compliquée de beaucoup d'autres, et il peut se faire qu'il y ait entre elles une compensation plus ou moins parfaite, comme celles dont les applications de la transformation de LORENTz nous ont déjà donné tant d'exemples.

B) On peut avoir

$$
\frac{t-\sum x \xi_{\mathrm{I}}}{\sqrt{\mathrm{I}-\sum \xi_{\mathrm{I}}^{2}}}=0, \quad t=\sum x \xi_{\mathrm{I}} .
$$

La vitesse de propagation est alors beaucoup plus rapide que celle de la lumière; mais dans certains cas $t$ pourrait être négatif, ce qui, comme nous l'avons dit, ne parait guère admissible. Nous nous en tiendrons donc à l'bypotbèse $(A)$.

$2^{\circ}$ Les quatre invariants $(7)$ doivent être des fonctions des invariants (5).

$3^{\circ}$ Quand les deux corps sont en repos absolu, $X_{1}, Y_{\mathrm{I}}, Z_{\mathrm{I}}$ doivent avoir la va- 
leur déduite de la loi de Newton, et quand ils sont en repos relatif, la valeur déduite des equations (4).

Dans l'hypothèse du repos absolu, les deux premiers invariants (7) doivent se réduire a

ou, par la loi de Newton, a

$$
\sum X_{1}^{2}, \quad \sum X_{1} x
$$

$$
\frac{\mathrm{I}}{r^{4}}, \quad-\frac{\mathrm{I}}{r} \text {; }
$$

d'autre part, dans l'hypothèse $(A)$, le $2^{\mathrm{d}}$ et le $3^{\mathrm{e}}$ des invariants $(5)$ deviennent:

$$
\frac{-r-\sum x \xi}{\sqrt{I-\sum \xi^{2}}}, \frac{-r-\sum x \xi_{\mathrm{I}}}{\sqrt{\mathrm{I}-\sum \xi_{\mathrm{I}}^{2}}},
$$

c'est-à-dire, pour le repos absolu, à

$$
-r, \quad-r \text {. }
$$

Nous pouvons donc admettre par exemple que les deux premiers invariants (4) se reduisent d

$$
\frac{\left(I-\sum \xi_{I}^{2}\right)^{2}}{\left(r+\sum x \xi_{1}\right)^{4}}, \quad-\frac{\sqrt{I-\sum \xi_{I}^{2}}}{r+\sum x \xi_{1}} ;
$$

mais d'autres combinaisons sont possibles.

Il faut faire un choix entre ces combinaisons, et, d'autre part, pour définir $X_{\mathrm{I}}$, $Y_{s}, Z_{\mathrm{r}}$ il nous faut une $3^{\mathrm{e}}$ équation. Pour un pareil choix, nous devons nous efforcer de nous rapprocher autant que possible de la loi de Newton. Voyons done ce qui se passe quand (faisant toujours $t=-r$ ) on néglige les carrés des vitesses $\xi$, $n$, etc. Les 4 invariants $(5)$ deviennent alors:

et les 4 invariants (7):

$$
0,-r-\sum x \xi,-r-\sum x \xi_{1}, \quad \text { I }
$$

$$
\sum X_{1}^{2}, \quad \sum X_{1}(x+\xi r), \quad \sum X_{1}\left(\xi_{1}-\xi\right), \quad 0 .
$$

Mais pour pouvoir comparer avec la loi de Newton, une autre transformation est nécessaire; ici $x_{0}+x, y_{0}+y, z_{0}+z$, représentent les coordonnées du corps attirant $d$ l'instant $t_{0}+t$, et $r=\sqrt{\sum x^{2}}$; dans la loi de Newton il faut envisager les coordonnées $x_{0}+x_{1}, y_{0}+y_{1}, z_{0}+z_{1}$ du corps attirant à l'instant $t_{0}$, et la distance $r_{\mathrm{r}}=\sqrt{\sum x_{\mathrm{I}}^{2}}$.

Nous pouvons négliger le carré du temps $t$ nécessaire à la propagation et par conséquent faire comme si le mouvement était uniforme; nous avons alors:

$$
\begin{gathered}
x=x_{1}+\xi_{1} t, \quad y=y_{1}+n_{I} t, \quad z=z_{1}+\zeta_{I} t \\
r\left(r-r_{1}\right)=\sum x \xi_{1} t ;
\end{gathered}
$$


ou, puisque $t=-r$,

$$
x=x_{\mathrm{I}}-\xi_{\mathrm{I}} r, \quad y=y_{\mathrm{r}}-n_{\mathrm{I}} r, \quad z=z_{\mathrm{I}}-\zeta_{\mathrm{I}} r, \quad r=r_{\mathrm{I}}-\sum x \xi_{\mathrm{r}} ;
$$

de sorte que nos 4 invariants $(5)$ deviennent:

$$
0, \quad-r_{1}+\sum x\left(\xi_{1}-\xi\right), \quad-r_{1}, \quad \text { I }
$$

et nos 4 invariants (7):

$$
\sum X_{1}^{2}, \quad \sum X_{\mathrm{I}}\left[x_{\mathrm{I}}+\left(\xi-\xi_{\mathrm{I}}\right) r_{\mathrm{I}}\right], \quad \sum X_{\mathrm{I}}\left(\xi_{\mathrm{I}}-\xi\right), \quad \text { o. }
$$

Dans la seconde de ces expressions j'ai écrit $r_{1}$ au lieu de $r$, parce que $r$ est multiplié par $\xi-\xi_{1}$ et que je néglige le carré de $\xi$.

D'autre part, la loi de Newton nous donnerait, pour ces 4 invariants (7),

$$
\frac{\mathrm{I}}{r_{1}^{4}}, \quad-\frac{\mathrm{I}}{r_{\mathrm{I}}}-\frac{\sum x_{\mathrm{I}}\left(\xi-\xi_{\mathrm{I}}\right)}{r_{1}^{2}}, \quad \frac{\sum x_{\mathrm{I}}\left(\xi-\xi_{\mathrm{I}}\right)}{r_{\mathrm{I}}^{3}}, \quad 0 .
$$

Si donc nous appelons $A$ et $B$, le $2^{\mathrm{d}}$ et le $3^{\mathrm{e}}$ des invariants (5), et $M, N, P$ les 3 premiers invariants (7), nous satisferons à la loi de Newton, aux termes près de l'ordre du carré des vitesses, en faisant:

$$
M=\frac{\mathrm{I}}{B^{4}}, \quad N=\frac{+A}{B^{2}}, \quad P=\frac{A-B}{B^{3}} .
$$

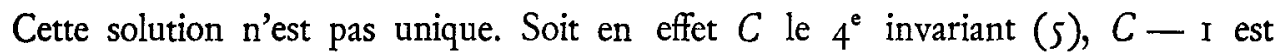
de l'ordre du carré de $\xi$, et il en est de même de $(A-B)^{2}$.

Nous pourrions donc ajouter aux $2^{\mathrm{ds}}$ membres de chacune des équations (8) un terme formé de $C$ - I multiplié par une fonction arbitraire de $A, B, C$ et un terme formé de $(A-B)^{2}$ multiplié également par une fonction de $A, B, C$.

$\mathrm{Au}$ premier abord, la solution (8) parait la plus simple, elle ne peut néanmoins être adoptée; en effet, comme $M, N, P$ sont des fonctions de $X_{1}, Y_{1}, Z_{1}$ et de $T_{1}=\sum X_{1} \xi$, on peut tirer de ces trois équations (8) les valeurs de $X_{1}, Y_{1}, Z_{1}$; mais dans certains cas ces valeurs deviendraient imaginaires.

Pour éviter cet inconvénient, nous opérerons d'une autre manière. Posons:

$$
k_{\mathrm{o}}=\frac{\mathrm{I}}{\sqrt{\mathrm{I}-\sum \xi^{2}}}, \quad k_{\mathrm{I}}=\frac{\mathrm{I}}{\sqrt{\mathrm{I}-\sum \xi_{\mathrm{r}}^{2}}},
$$

ce qui est justifié par l'analogie avec la notation

$$
k=\frac{\mathrm{I}}{\sqrt{\mathrm{I}-\varepsilon^{2}}}
$$

qui figure dans la substitution de LoRentz.

Dans ce cas, et $\mathrm{a}$ cause de la condition $-r=t$, les invariants ( 5 ) deviennent :

$$
\text { o, } A=-k_{\mathrm{o}}\left(r+\sum x \xi\right), \quad B=-k_{\mathrm{I}}\left(r+\sum x \xi_{\mathrm{I}}\right), \quad C=k_{\mathrm{o}} k_{\mathrm{I}}\left(\mathrm{I}-\sum \xi \xi_{\mathrm{s}}\right) \text {. }
$$


D'autre part, nous voyons que les systèmes suivants de quantités:

$$
\begin{array}{cccc}
x, & y, & z, & -r=t \\
k_{\mathrm{o}} X_{\mathrm{I}}, & k_{\mathrm{o}} Y_{\mathrm{r}}, & k_{\mathrm{o}} Z_{\mathrm{I}}, & k_{\mathrm{o}} T_{\mathrm{I}} \\
k_{\mathrm{o}} \xi, & k_{\mathrm{o}} n_{1} & k_{\mathrm{o}} \zeta, & k_{\mathrm{o}} \\
k_{\mathrm{I}} \xi_{\mathrm{I}}, & k_{\mathrm{I}} n_{\mathrm{I}}, & k_{\mathrm{I}} \zeta_{\mathrm{I}}, & k_{\mathrm{I}}
\end{array}
$$

subissent les mêmes substitutions linéaires quand on leur applique les transformations du groupe de Lorentz. Nous sommes donc conduits à poser:

(9)

$$
\left\{\begin{array}{l}
X_{\mathrm{I}}=x \frac{\alpha}{k_{\mathrm{o}}}+\xi \beta+\xi_{\mathrm{I}} \frac{k_{\mathrm{I}}}{k_{\mathrm{o}}} \gamma, \\
Y_{\mathrm{r}}=y \frac{\alpha}{k_{\mathrm{o}}}+\eta \beta+\eta_{\mathrm{I}} \frac{k_{\mathrm{I}}}{k_{\mathrm{o}}} \gamma, \\
Z_{\mathrm{s}}=z \frac{\alpha}{k_{\mathrm{o}}}+\zeta \beta+\zeta_{\mathrm{I}} \frac{k_{\mathrm{I}}}{k_{\mathrm{o}}} \gamma, \\
T_{\mathrm{I}}=-r \frac{\alpha}{k_{\mathrm{o}}}+\beta+\frac{k_{\mathrm{I}}}{k_{\mathrm{o}}} \gamma .
\end{array}\right.
$$

Il est clair que si $\alpha, \beta, \gamma$ sont des invariants, $X_{1}, Y_{1}, Z_{1}, T_{1}$ satisferont d la condition fondamentale, c'est-d-dire subiront, par l'effet de transformations de LoRENTz, une substitution linéaire convenable.

Mais pour que les équations (9) soient compatibles, il faut que l'on ait:

$$
\sum X_{\mathrm{r}} \xi-T_{\mathrm{s}}=0,
$$

ce qui, en remplaçant $X_{1}, Y_{1}, Z_{1}, T_{1}$ par leurs valeurs (9) et en multipliant par $k_{\circ}^{2}$, devient:

$$
-A \alpha-\beta-C_{\gamma}=0 .
$$

Ce que nous voulons, c'est que si l'on néglige, devant le carré de la vitesse de la lumière, les carrés des vitesses $\xi$, etc., ainsi que le produit des accélérations par les distances, comme nous l'avons fait plus haut, les valeurs de $X_{\mathrm{I}}, Y_{\mathrm{I}}, Z_{\mathrm{I}}$ restent conformes à la loi de Newton.

Nous pourrons prendre:

$$
\beta=0, \quad \gamma=-\frac{A x}{C} .
$$

Avec l'ordre d'approximation adopté, on a:

$$
\begin{gathered}
k_{\mathrm{o}}=k_{\mathrm{x}}=\mathrm{I}, \quad C=\mathrm{I}, \quad A=-r_{\mathrm{I}}+\sum x\left(\xi_{\mathrm{r}}-\xi\right), \quad B=-r_{\mathrm{r}}, \\
x=x_{\mathrm{r}}+\xi_{\mathrm{I}} t=x_{\mathrm{r}}-\xi_{\mathrm{r}} r .
\end{gathered}
$$

La I ére équation (9) devient alors:

$$
X_{\mathrm{s}}=\alpha\left(x-A \xi_{\mathrm{s}}\right) \text {. }
$$

Mais si on néglige le carré de $\xi$, on peut remplacer $A \xi_{\mathrm{I}}$ par $-r_{\mathrm{I}} \xi_{\mathrm{I}}$ ou encore 
par $-r \grave{i}_{1}$, ce qui donne:

$$
X_{1}=\alpha\left(x+\xi_{1} r\right)=\alpha x_{1} .
$$

La loi de Newton donnerait

$$
X_{x}=-\frac{x_{t}}{r_{1}^{3}}
$$

Nous devons donc choisir, pour l'invariant $\alpha$, celui qui se réduit $\grave{a}-\frac{\mathrm{I}}{r_{\mathrm{I}}^{3}} \grave{d}$ l'ordre d'approximation adopté, c'est-à-dire $\frac{\mathrm{I}}{B^{3}}$. Les équations (9) deviendront:

(II)

$$
\left\{\begin{array}{l}
X_{\mathrm{r}}=\frac{x}{k_{\mathrm{o}} \bar{B}^{3}}-\xi_{\mathrm{I}} \frac{k_{\mathrm{I}}}{k_{\mathrm{o}}} \frac{A}{B^{3} C}, \\
Y_{\mathrm{I}}=\frac{y}{k_{\mathrm{o}} \bar{B}^{3}}-\eta_{\mathrm{I}} \frac{k_{\mathrm{I}}}{k_{\mathrm{o}}} \frac{A}{B^{3} C}, \\
Z_{\mathrm{r}}=\frac{z}{k_{\mathrm{o}} B^{3}}-\zeta_{\mathrm{I}} \frac{k_{\mathrm{I}}}{k_{\mathrm{o}}} \frac{A}{B^{3} C}, \\
T_{\mathrm{r}}=-\frac{r}{\overline{k_{\mathrm{o}} B^{3}}}-\frac{k_{\mathrm{I}}}{k_{\mathrm{o}}} \frac{A}{B^{3} C} .
\end{array}\right.
$$

Nous voyons d'abord que l'attraction corrigée se compose de deux composantes; l'une parallèle au vecteur qui joint les positions des deux corps, l'autre parallèle à la vitesse du corps attirant.

Rappelons que quand nous parlons de la position ou de la vitesse du corps attirant, il s'agit de sa position ou de sa vitesse au moment où l'onde gravifique le quitte; pour le corps attiré, au contraire, il s'agit de sa position ou de sa vitesse du moment où l'onde gravifique l'atteint, cette onde étant supposée se propager avec la vitesse de la lumière.

Je crois qu'il serait prématuré de vouloir pousser plus loin la discussion de ces formules; je me bornerai done à quelques remarques.

$\mathrm{I}^{\circ}$ Les solutions (I I) ne sont pas uniques; on peut, en effet, remplacer $\frac{I}{B^{3}}$, qui entre en facteur partout, par

$$
\frac{\mathrm{I}}{B^{3}}+(C-\mathrm{I}) f_{\mathrm{1}}(A, B, C)+(A-B)^{2} f_{2}(A, B, C),
$$

$f_{1}$ et $f_{2}$ étant des fonctions arbitraires de $A, B, C$, ou encore ne plus prendre $\beta$ nul, mais ajouter $\grave{a} \alpha, \beta, \gamma$ des termes complémentaires quelconques, pourvu qu'ils satisfassent d la condition (IO) et qu'ils soient du $2^{\mathrm{d}}$ ordre par rapport aux $\xi$, en ce qui concerne $\alpha$, et du $\mathrm{I}^{\text {er }}$ ordre en ce qui concerne $\beta$ et $\gamma$.

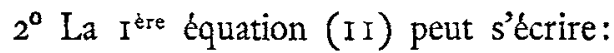

$$
X_{1}=\frac{k_{\mathrm{r}}}{B^{3} C}\left[x\left(\mathrm{I}-\sum \xi \xi_{\mathrm{I}}\right)+\xi_{\mathrm{I}}\left(r+\sum x \xi\right)\right]
$$

et la quantité entre crochets peut, elle-même, s'ecrire :

$$
\left(x+r \xi_{\mathrm{r}}\right)+n\left(\xi_{\mathrm{I}} y-x \eta_{\mathrm{s}}\right)+\zeta_{\left(\xi_{\mathrm{I}} z-x \zeta_{\mathrm{s}}\right)}
$$


de sorte que la force totale peut itre partagie en trois composantes correspondant aux trois parenthéses de l'expression (I2); la première composante a une vague analogie avec la force mécanique due au champ électrique, les deux autres arec la force mécanique due au champ nagnéétique; pour compléter l'analogie je puis, en vertu de la İere remarque, remplacer dans les équations (II) $\frac{1}{B^{3}}$, par $\frac{C}{B^{3}}$, de façon que $X_{1}, Y_{1}, Z_{1}$ ne dépendent plus que linéairement de la vitesse $\check{c}, n, \zeta$ du corps attiré, puisque $C$ a disparu du dénominateur de ( $\left.\mathrm{I}^{\mathrm{bis}}\right)$.

Posons alors:

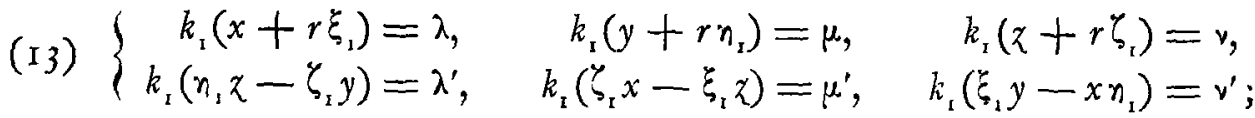

il viendra, $C$ ayant disparu du dénominateur de $\left(I^{\text {bis }}\right)$ :

$$
\left\{\begin{array}{l}
X_{\mathrm{z}}=\frac{\lambda}{B^{3}}+\frac{\eta \nu^{\prime}-\zeta \mu^{\prime}}{B^{3}}, \\
Y_{\mathrm{x}}=\frac{\mu}{B^{3}}+\frac{\zeta \lambda^{\prime}-\xi \nu^{\prime}}{B^{3}}, \\
Z_{\mathrm{x}}=\frac{\nu}{B^{3}}+\frac{\xi \mu^{\prime}-n \lambda^{\prime}}{B^{3}} ;
\end{array}\right.
$$

et on aura d'ailleurs:

$$
B^{2}=\sum \lambda^{2}-\sum \lambda^{\prime 2}
$$

Alors $\lambda, \mu, v$, ou $\frac{\lambda}{B^{3}}, \frac{\mu}{B^{3}}, \frac{v}{B^{3}}$, est une espèce de champ électrique, tandis que $\lambda^{\prime}, \mu^{\prime}, \nu^{\prime}$, ou plutôt $\frac{\lambda^{\prime}}{\bar{B}^{3}}, \frac{\mu^{\prime}}{B^{3}}, \frac{v^{\prime}}{\overline{B^{3}}}$, est une espèce de champ magnétique.

$3^{\circ}$ Le postulat de relativité nous obligerait à adopter la solution (II) ou la solution (I4) ou l'une quelconque des solutions qui s'en déduiraient à l'aide de la I $^{\text {ire }}$ remarque; mais la première question qui se pose est celle de savoir si elles sont compatibles avec les observations astronomiques; la divergence avec la loi de Newton est de l'ordre de $\xi^{2}, c^{\prime}$ est-t-dire 10000 fois plus' petite que si elle était de l'ordre de $\xi$, c'est-d̀-dire si la propagation se faisait avec la vitesse de la lumière, ceteris non mutatis; il est donc permis d'espérer qu'elle ne sera pas trop grande. Mais une discussion approfondie pourra seule nous l'apprendre.

Paris, juillet rgos.

H. Poincaré. 